(C) 1997 International Press

Adv. Theor. Math. Phys. 1 (1997) 388-429

\title{
Quantum Theory of Geometry II: Volume operators
}

\author{
Abhay Ashtekar ${ }^{a, d}$, Jerzy Lewandowski ${ }^{b, c, d}$ \\ ${ }^{a}$ Center for Gravitational Physics and Geometry \\ Department of Physics, Penn State, \\ University Park, PA 16802-6300, USA \\ ${ }^{b}$ Instytut Fizyki Teoretycznej, Uniwersytet Warszawski \\ ul. Hoza 69, 00-681 Warszawa, Poland \\ ${ }^{c}$ Max-Planck-Institut für Gravitationsphysik, Albert Einstein Institut, \\ Schlaatzweg 1, D-14473 Potsdam, Germany \\ ${ }^{d}$ Erwin Schrödinger International Institute for Mathematical Sciences \\ Boltzmanngasse 9, A-1090 Vienna, Austria
}

\begin{abstract}
A functional calculus on the space of (generalized) connections was recently introduced without any reference to a background metric. It is used to continue the exploration of the quantum Riemannian geometry. Operators corresponding to volume of three-dimensional regions are introduced rigorously. It is shown that there are two natural regularization schemes, each of which leads to a well-defined operator. Both operators can be completely specified by giving their action on states labelled by graphs. The two final results are closely related but differ from one another in that one of the operators is sensitive to the differential structure of graphs at their vertices while the second is sensitive only to the topological characteristics. (The second operator was first introduced by Rovelli and Smolin and De Pietri and Rovelli using a somewhat different framework.) The difference between the two operators can be attributed directly to the standard quantization ambiguity. Underlying assumptions and subtleties of regularization procedures are discussed in detail in both cases because volume operators play an important role in the current discussions of quantum dynamics.
\end{abstract}




\section{Introduction}

Riemannian geometry provides the mathematical framework for general relativity and other modern theories of gravity. One therefore expects that a non-perturbative formulation of quantum gravity would require a corresponding quantum theory of geometry, and, at the same time, provide pointers for constructing this theory. Familiar Riemannian geometry would then emerge only as an approximation on coarse graining of the semi-classical states. In a specific non-perturbative approach based on canonical quantization, these expectations are being borne out in detail. The goal of this series of papers is to present the resulting quantum theory of geometry. Basic techniques used in this series were developed in [1] and applied to the problem of constructing area operators. The purpose of this paper is to carry out a similar construction of volume operators.

Let us begin with a brief summary. In the canonical quantization approach used here, the configuration variable is an $S U(2)$ connection $A_{a}^{i}(x)$ on a three-manifold $\Sigma$. (Indices $a, b, c, \ldots$ refer to the tangent space of $\Sigma$ and indices $i, j, k, \ldots$ are the $s u(2)$ Lie-algebra indices.) The momentum variable is a vector density $E_{i}^{a}(x)$ with values in the $s u(2)$ Lie algebra (or, equivalently, a (pseudo) two-form $e_{a b i}:=\eta_{a b c} E_{i}^{c}$, where $\eta_{a b c}$ is the LeviCivita pseudo density). In the quantum theory, then, one is naturally led to consider the space $\overline{\mathcal{A}}$ of (suitably generalized) connections on $\Sigma$ as the (quantum) configuration space. To obtain the Hilbert space $\mathcal{H}$ of quantum states and geometric operators thereon, one needs a functional calculus on $\overline{\mathcal{A}}$ which also does not refer to a fiducial metric (or any other background field).

The necessary tools were developed in a series of papers by a number of authors [2-11]. (Much of the motivation for this work came from the 'loop representation' introduced earlier by Rovelli and Smolin [12].) It turns out that $\overline{\mathcal{A}}$ admits a natural diffeomorphism invariant measure $\mu^{\circ}$ and the Hilbert space $\mathcal{H}$ can be taken to be the space $L^{2}\left(\overline{\mathcal{A}}, d \mu^{o}\right)$ of square-integrable functions on $\overline{\mathcal{A}}[3-8]$. Physically, $\mathcal{H}$ represents the space of kinematic quantum states, i.e., the quantum analog of the full phase space. Using the well-developed differential geometry on $\overline{\mathcal{A}}$ [7], one can then define physically interesting operators on $\mathcal{H}$. In particular, one can introduce, in a systematic manner, operator-valued distributions $\hat{E}$ corresponding to the triads [1]. As in classical Riemannian geometry, these are the basic objects in the quantum case. Specifically, the idea is to construct geometric operators -e.g., those corresponding to area, volume and length- by regularizing the appropriate products of these triad operators.

As remarked above, triads -being density weighted- can be naturally thought of as pseudo two-forms $e_{a b i}$. To obtain phase space functions, it is 
natural to smear them against Lie-algebra-valued test fields $f_{i}$ with support on two-dimensional surfaces. Can the corresponding quantum operators be well-behaved? The answer is not a priori obvious: from Minkowskian quantum field theory, one would expect that well-defined operators will not result unless they are smeared in (at least) three dimensions. Somewhat surprisingly, however, for triads the answer is in the affirmative. More generally, in this approach to quantum geometry, there is a remarkable synergy between geometry and analysis: in the regularization procedure, well-defined operators result when $n$-forms are integrated on $n$-manifolds. Thus, the operators that code information in connections are holonomies $\hat{h}[\alpha]$, obtained by integrating the connection one-forms along one-dimensional curves. The two-form triad operators are naturally regulated through a two-dimensional smearing. This feature is deeply intertwined with the underlying diffeomorphism invariance of the theory. By contrast, in the quantum theory of Maxwell fields in Minkowski space-time, for example, one smears both connection one-forms and electric field two-forms in three dimensions, using the geometrical structures made available by the background metric.

In [1], square-roots of appropriate products of triad operators were regularized to obtain area operators $\hat{A}_{S}$ associated with two-dimensional surfaces $S$ without boundary. These are the quantum analogs of the area functions $A_{S}$ defined on the classical phase space. We now wish to discuss the volume operator $\hat{V}_{R}$ associated with a three-dimensional region $R$ - the quantum analog of the function $V_{R}:=\int_{R} d^{3} x \sqrt{|\operatorname{det} E|}$ on the classical phase space. Since $V_{R}$ is a rather complicated, non-polynomial function of the triads, as one might imagine, the issue of regularization is quite subtle. Indeed, the problem turns out to be considerably more complicated than that for area operators. In particular (for the continuum theory) it appears that, so far, no regularization scheme has appeared in the literature which has the following rather basic property: If we denote by $\hat{V}_{R}^{\epsilon}$ the regulated version of the volume operator, then (in the Hilbert space topology) the $\operatorname{limit}_{\epsilon \rightarrow 0}\left(\hat{\lim }_{R}^{\epsilon} \cdot \Psi\right)$ should exist for a dense subset of states $\Psi \in \mathcal{H}$. We will rectify this situation. (In the process, we also spell out the underlying assumptions and point out some subtleties that are often overlooked.) Finally, in our treatment, the limit is achieved at a finite stage, i.e., for all $\epsilon \leq \epsilon \Psi$. These properties make our regularization of volume operators consistent with that of the Hamiltonian constraint; there is thus a uniform scheme that is applicable to all operators of physical interest. ${ }^{1}$

In the main body of the paper, we will discuss a regularization along the lines that led us to area operators in [1]. We will see that this operator is sensitive to the differential structure on the three-manifold $\Sigma$. In the appendix, we will consider a different regularization scheme which is based on

\footnotetext{
${ }^{1}$ For a further discussion of this point, see the first part of the Appendix.
} 
a construction given by Rovelli and Smolin [13] in the loop representation. In a certain sense, this operator is not sensitive to the differential structure of $\Sigma$. We will see that both operators can be constructed through systematic regularizations. They are well-defined, self-adjoint operators on $\mathcal{H}$ with purely discrete spectrum. The actual expressions of the two operators are rather similar and the difference between them can be interpreted simply as a 'quantization ambiguity'. Nonetheless, in various applications, e.g., to quantum dynamics [14], they can lead to important differences. To get a deeper understanding of the relation between them, one needs to further analyze their properties -e.g., their relation to the area and length operators. Such a systematic analysis has begun only recently.

Several researchers have worked on geometric operators. A significant fraction of this work was presented only in seminars and informal discussions and is therefore difficult to document. The situation with the published material may be briefly summarized as follows. The simplest calculations, which are rather naive but nonetheless capture the germ of the basic idea, were first reported in [15]. A more detailed regularization procedure was developed in the 'multi-loop framework' by Smolin [16] and applied, in particular, to area and volume operators. The final expression of this volume operator is closely related to that obtained in the main part of the present paper. Rovelli and Smolin [13] analyzed both area and volume operators in terms of spin networks. They found a part of the spectrum of area operators and gave, in the loop representation, an explicit expression of the volume operator on trivalent graphs. However, it was later realized that there was a computational error and that the correct volumeoperator in fact annihilates these trivalent states. The final closed-form expression of this operator was introduced by De Pietri and Rovelli in [17], and, in the framework discussed here, in [18]. A comprehensive treatment of area operators -including the complete spectrum- was given in [1] using the Hilbert space $\mathcal{H}$ of generalized connections and the complete spectrum was then re-derived in the loop representation by Frittelli, Lehner and Rovelli [19]. The final form of the volume operator derived here in the main text was reported in $[7,18,20]$ and its restriction to a lattice theory was discussed by Loll [21]. The operator was also studied by Thiemann in [22]. Finally, the length operator was introduced by Thiemann in [23].

This paper is organized as follows. Section 2 is devoted to preliminaries. Regularization leading to the first operator is discussed in detail in Sections 3 and 4 and, using techniques introduced by De Pietri [24], the regularization leading to the Rovelli-Smolin operator is discussed in the Appendix. Some properties of the volume operator are discussed in Section 5. Section 6 summarizes the main results and compares the regularization procedures.

For simplicity, in the main discussion, we have set $c=1,8 \pi G=1$ and 
$\hbar=1$ and chosen the real connection $A_{a}^{i}$ to be $A_{a}^{i}=\Gamma_{a}^{i}-K_{a}^{i}$ where $\Gamma_{a}^{i}$ is the spin connection compatible with the triad $E_{i}^{a}$ and $K_{a}^{i}$ is the extrinsic curvature. As pointed out by Immirzi [32] using earlier work of Barbero [31], unitarily inequivalent quantum theories result if one begins with the canonical pair ${ }^{\gamma} A_{a}^{i}=\Gamma_{a}^{i}-\gamma K_{a}^{i},{ }^{\gamma} E_{i}^{a}=(1 / \gamma) E_{i}^{a}$, where $\gamma$ is a non-zero real parameter. Thus, in the main discussion, we will work with the $\gamma=1$ sector. In Section 4.4, we will restore $c, \hbar$ and $G$ and also state the main result in any Immirzi sector.

\section{Preliminaries}

In this section, we briefly recall the mathematical ideas that underlie the present approach to quantum Riemannian geometry. This discussion will also serve to fix notation. It turns out that some diversity has arisen in viewpoints and conventions in the recent literature on non-perturbative quantum gravity. To remove potential confusion, therefore, the corresponding issues will be discussed in detail.

Fix an orientable, analytic ${ }^{2}$ three-manifold $\Sigma$ and a principal $S U(2)$ bundle $B$ over $\Sigma$. Our configuration space $\mathcal{C}$ will consist of smooth connections on $B$. Since all $S U(2)$ bundles over three-manifolds are trivial, we can fix a trivialization and regard each connection $A$ on $B$ as an $s u(2)$-valued oneform $A_{a}^{i}$ on $\Sigma$, where $a$ is the form index, and $i$ the Lie-algebra index. (We will not specify boundary conditions on fields because they are irrelevant for the issues we wish to discuss here.) The 'conjugate momenta' are nondegenerate vector densities $E_{i}^{a}$ of weight one - or, equivalently, pseudo twoforms $e_{a, b i}:=\eta_{a b c} E_{i}^{c}$ - with values in $s u(2)$, where $\eta^{a b c}$ is the Levi-Civita pseudo density. Thus, the action

$$
\int_{\Sigma} d^{3} x E_{i}^{a} \delta A_{a}^{i} \equiv \int_{\Sigma} \delta A^{i} \wedge e_{i}
$$

of the cotangent vector $E_{i}^{a}$ on a tangent vector $\delta A_{a}^{i}$ is invariant under the change of orientation of $\Sigma$. Although they are density weighted, for brevity, the $E_{i}^{a}$ will be referred to simply as triads.

Riemannian geometry of the three-manifold $\Sigma$ is coded in the momenta $E_{i}^{a}$. To see this, note first that given vector densities $E_{i}^{a}$, we can define a triplet of vector fields $e_{i}^{a}$ via:

$$
e_{i}^{a}=\frac{E_{i}^{a}}{\sqrt{|\operatorname{det} E|}},
$$

\footnotetext{
${ }^{2}$ In this work the assumption of analyticity is not essential; we make it for simplicity since it allows us to use previous results $[1-4,6-9]$ directly. Our constructions can be made to go through for smooth manifolds and graphs. This point is discussed at the end of section 4.
} 
where $\operatorname{det} E$ stands for the determinant of the matrix $\left(E^{i a}\right)$ with $i, a=1,2,3$. Note that the phase space contains frame fields $e_{i}^{a}$ with both orientations. Given these fields, we can just define a contravariant, positive definite metric $q^{a b}:=e_{i}^{a} e_{j}^{b} k^{i j}$ where $k=-2 \operatorname{Tr}$ is the Cartan-Killing metric on $s u(2)$. If we denote by $q$ the determinant of $q_{a b}$ (the inverse of $q^{a b}$ ), we also have: $E_{i}^{a}=\sqrt{q} e_{i}^{a} \equiv|\operatorname{det} e| e_{i}^{a}$. In terms of these Riemannian structures, the volume of a region $R$ (covered, for simplicity, by a single chart) is given by:

$$
V_{R}:=\int_{R} d^{3} x \sqrt{q} \equiv \int_{R} d^{3} x \sqrt{|\operatorname{det} E|}
$$

As noted in the Introduction, in quantum theory one is naturally led $[2,3]$ to consider the space $\overline{\mathcal{A}}$ of (suitably generalized) connections as the configuration space. Thus, the Hilbert space $\mathcal{H}$ of (kinematic) quantum states is given by $\mathcal{H}=L^{2}\left(\overline{\mathcal{A}}, d \mu^{o}\right)$ where $\mu^{o}$ is a natural diffeomorphism invariant measure on $\overline{\mathcal{A}}[3-8]$. $\mathcal{H}$ contains a dense subspace Cyl of 'cylindrical functions' which turns out to be especially useful. These are constructed as follows. Each element $\bar{A}$ of $\overline{\mathcal{A}}$ assigns to any oriented analytic path $p$ in $\Sigma$ an element $\bar{A}(p)$ of $S U(2)$ (which can be regarded as the 'holonomy' of the generalized connection $\bar{A}$ ) [7]. Fix a graph $\gamma$ with a finite number (say $N$ ) of edges $^{3} e_{I}, I=1, \ldots, N$, and a complex-valued function $\psi:[S U(2)]^{N} \rightarrow \mathbf{C}$ on $[S U(2)]^{N}$. Then, we can define a function on $\overline{\mathcal{A}}$ as follows:

$$
\Psi_{\gamma}(\bar{A})=\psi\left(\bar{A}\left(e_{1}\right), \ldots, \bar{A}\left(e_{N}\right)\right) .
$$

(Strictly, the function on the left side should be written as $\Psi_{\gamma, \psi}$. However, for notational simplicity, we will omit the subscript $\psi$.) Note that $\Psi_{\gamma}$ 'knows' only about what the connection $\bar{A}$ does on the $N$ edges of $\gamma$; it depends only on a finite number of 'coordinates' on $\overline{\mathcal{A}}$. Therefore, following standard terminology, $\Psi_{\gamma}$ are called cylindrical functions. The space of cylindrical functions defined by a fixed graph $\gamma$ is infinite dimensional but 'rather small' in the sense that it can be thought of as the space of quantum states of a system with only a finite number of degrees of freedom. However, as we vary $\gamma$ through all finite graphs on $\Sigma$, we obtain a space of functions on $\overline{\mathcal{A}}$ which is very large. This is the space Cyl. Since it is dense in $\mathcal{H}$, we can first define physically interesting operators on Cyl and then consider their self-adjoint extensions. This strategy turns out to be especially convenient in practice.

Of special interest to us are 'angular momentum like' operators $J_{x, e}^{i}$, associated with a point $x$ in $\Sigma$ and an edge $e$ which begins at $x$, where, as

\footnotetext{
${ }^{3}$ By a graph $\gamma$ we mean a finite set $\left\{e_{1}, \ldots, e_{N}\right\}$ of oriented one-dimensional, analytic sub-manifolds of $\Sigma$ such that each $e_{I}$ has a (two point) boundary and for $I_{1} \neq I_{2}$ the intersection $e_{I_{1}} \cap e_{I_{2}}$ is contained in the boundaries. The elements $e_{I}$ of a graph are called edges, the points in their boundaries the vertices.
} 


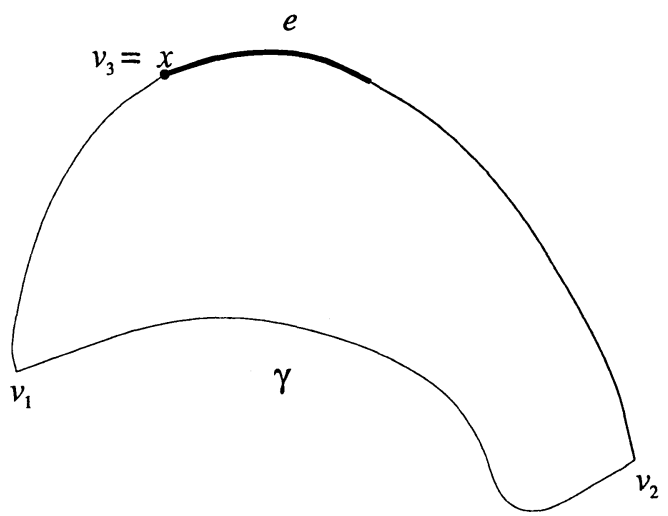

Figure 1: Illustration of the action of $J_{x, e}^{i}$ operator on $\Psi_{\gamma}$.

before, $i$ is an $s u(2)$ index. Given $\Psi_{\gamma}$ which can be regarded as cylindrical i.e., represented as in (2.3) - with respect to a graph which does not contain a segment of $e$ with $x$ as one of its end points,

$$
\left(J_{x, e}^{i} \Psi_{\gamma}\right)(\bar{A})=0 .
$$

If on the other hand $\gamma$ has an edge $e_{J}$ which shares a finite segment with $e$ originating at $x$, without loss of generality, we can assume that $x$ is a vertex of $\gamma$. Then,

$$
\begin{aligned}
\left(J_{x, e}^{i} \Psi_{\gamma}\right)(\bar{A}) & =i\left(\bar{A}\left(e_{J}\right) \tau^{i}\right)_{B}^{A} \frac{\partial \psi}{\partial\left(\bar{A}\left(e_{J}\right)\right)_{B}^{A}} \\
& =i \frac{d}{d t}\left[\psi\left(\bar{A}\left(e_{1}\right), \ldots, \bar{A}\left(e_{J}\right) \exp \left(t \tau^{i}\right), \ldots, \bar{A}\left(e_{N}\right)\right]\right.
\end{aligned}
$$

if $e_{J}$ is outgoing at $x .^{4}$ Here, $\tau^{i}$ are $s u(2)$ matrices, satisfying

$$
-2 \operatorname{Tr} \tau^{i} \tau^{j}=k^{i j} \quad \text { and }\left[\tau^{i}, \tau^{j}\right]=\epsilon^{i j k} \tau^{k},
$$

where $k_{i j}$ is the Killing form on $S U(2)$.

These operators are well-defined: If an element of Cyl is cylindrical with respect to two distinct graphs $\gamma$ and $\gamma^{\prime}, \Psi_{\gamma^{\prime}}=\Psi_{\gamma}$, then $J_{x, e}^{i} \Psi_{\gamma^{\prime}}=J_{x, e}^{i} \Psi_{\gamma}$. Denote by $\mathrm{Cyl}^{(1)}$ the space of all cylindrical functions on $\overline{\mathcal{A}}$ for which $\psi$ in (2.3) is $C^{1}$. One can show that $J_{x, e}^{i}$ is essentially self-adjoint on the domain $\mathrm{Cyl}^{(1)}$. (For general results on essential self-adjointness of such operators,

\footnotetext{
${ }^{4}$ The operator on the right side of $(2.5)$ is just the (Lie derivative by the) left-invariant vector field $L^{i}$ of the copy of $S U(2)$ associated with the $J$-th edge. It is in this sense that $J_{x, e}^{i}$ are angular momentum-like operators. If $x$ is the end-point of the edge $e_{J}$ rather than the beginning-point, $L_{i}$ should be replaced by the right invariant vector field $-R^{i}$. For details, see Eq. (3.9) - (3.11) in Ref. [1].
} 
See [7].) Next, given a point $x \in \Sigma$ let us introduce an equivalence relation for the edges sharing $x$ as one of the ends: $e \sim e^{\prime}$ if and only if there is a neighborhood of $x$ on which $e$ and $e^{\prime}$ overlap modulo orientation. Then, $J_{x, e}^{i}=J_{x^{\prime}, e^{\prime}}^{i^{\prime}}$ if and only if $x=x^{\prime}, i=i^{\prime}$ and $e \sim e^{\prime}$. Finally, the commutation relations between these operators are given by:

$$
\left[J_{x, e}^{i}, J_{x^{\prime}, e^{\prime}}^{j}\right]=i \delta_{x, x^{\prime}} \delta_{[e],\left[e^{\prime}\right]} \epsilon_{k}^{i j} J_{x, e}^{k},
$$

where $[e]$ denotes the equivalence class of edges defined by the above equivalence relation and $\epsilon^{i j}{ }_{k}$ are the structure constants of $s u(2)$. In what follows, $[e]$ will be referred to as the germ of an edge $e$ starting at $x$.

Finally, let us recall from [1] the definition of the smeared triad operators, the quantum analogs of the classical expressions $E_{S}^{i}:=\frac{1}{2} \int_{S} \eta_{a b c} E^{i} d x^{a} \wedge d x^{b}$, where $S$ is an analytic two-surface without boundary. (Note that the action of $E_{S}^{i}$ depends on the relative orientation of $S$ and $\Sigma$ which naturally defines the notion of 'up' and 'down' with respect to $S$ ). A careful regularization leads to the following result:

$$
\hat{E}_{S}^{i}=\frac{1}{2} \sum_{x \in S} \sum_{[e]} \kappa_{S}([e]) J_{x, e}^{i},
$$

where the first sum ranges over all the points $x$ of $S$ and, for every $x,[e]$ runs through the set of germs starting at $x$, and where

$$
\kappa_{S}([e])= \begin{cases}1, & \text { if } e \text { lies above } S \\ -1, & \text { if } e \text { lies below } S \\ 0, & \text { otherwise }\end{cases}
$$

The sums in (2.7) are infinite. However, when we act with the right side on any cylindrical function, the result is well-defined because the sums have only a finite number of non-vanishing terms. Given a function $\Psi \in \mathrm{Cyl}$ it is convenient to represent it by (2.3) using a graph $\gamma$ such that every isolated intersection point between the range of $\gamma$ and $S$ is a vertex of $\gamma$. Then, the action of $\hat{E}_{S}^{i}$ on $\Psi=\Psi_{\gamma}$ reads

$$
\hat{E}_{S}^{i} \Psi_{\gamma}=\frac{1}{2} \sum_{v} \sum_{I} \kappa_{S}\left(\left[e_{I}\right]\right) J_{v, e_{I}}^{i} \Psi_{\gamma},
$$

where $v$ runs through the vertices of $\gamma$ contained in $S$, and $I$ through the (labels of) the edges of $\gamma$ intersecting $v$. The operator

$$
\hat{E}_{S}^{i}: \mathrm{Cyl}^{(1)}(\overline{\mathcal{A}}) \rightarrow \mathrm{Cyl}^{(0)}(\overline{\mathcal{A}})
$$

turns out to be essentially self-adjoint [1].

This concludes our discussion of mathematical preliminaries. In the remainder of the paper, the operators $J_{x, e}^{i}$ and $\hat{E}_{S}^{i}$ on $\mathrm{Cyl}^{(1)}$ will be used repeatedly. 


\section{3 'Internal' or 'Intrinsic' Regularization}

We can now introduce the first regularization of the volume operators. This discussion will be divided into three parts. In the first, we consider the classical theory and introduce an approximate expression of the volume functional by dividing the region under consideration into small cells. In the second, we take over this 'regulated' expression to the quantum theory. In the last part, we let the cells shrink and show that the limit yields a well-defined operator. However, this operator carries a memory of the background structure (namely, coordinates) introduced in the intermediate steps. To obtain a covariant operator, we need to 'average' over the relevant background structures. The averaging procedure is carried out in Section 4.

Fix an open region $R$ in $\Sigma$. We wish to construct the operator $\hat{V}_{R}$ corresponding to the function

$$
V_{R}(E):=\int_{R} d^{3} x|\operatorname{det} E|^{\frac{1}{2}}
$$

on the classical phase space. For the regularization procedure, it will be necessary to assume that $R$ can be covered by a single coordinate system $\left(x^{a}\right)$. However, it turns out that this assumption is not overly restrictive. To see this, note first that given any region $R$, we can cover it by a family $\mathcal{U}$ of neighborhoods such that each $U \in \mathcal{U}$ is covered by a single coordinate system. Let $\left(\phi_{U}\right)_{U \in \mathcal{U}}$ be a partition of unity associated to $\mathcal{U}$. Then, if we set

$$
V_{R, \phi_{U}}=\int_{R} d^{3} x \phi_{U}|\operatorname{det} E|^{\frac{1}{2}},
$$

we have

$$
V_{R}=\sum_{U \in \mathcal{U}} V_{R, \phi_{U}}
$$

where the final answer is independent of the specific choice of the partition of unity. It turns out that the same reasoning holds for the quantum operator $\hat{V}_{R}$. Hence, it will suffice to define the volume operators for regions $R$ which can be covered by a single coordinate system.

\subsection{Classical Basis for the Regularization Pprocedure}

Fix global coordinates $x^{a}$ in a neighborhood in $\Sigma$ containing $R$ and cover it with a family $\mathcal{C}$ of closed cubes whose sides are parallel to the coordinate planes. (If a cube $C$ is not contained in $R$, consider only its intersection with $R$.) Two different cells can share only points in their boundaries. Within 
each cell $C \in \mathcal{C}$ consider an ordered triple $s=\left(S_{1}, S_{2}, S_{3}\right)$ of oriented twosurfaces (without boundary) defined by

$$
x^{a}=\text { const }^{a}, \quad a=1,2,3,
$$

which intersect in the interior of $C$ (and whose orientation is induced by that of the coordinate axes). Such a family of pairs $(C, s)$, of cells and triples of surfaces $s$ ('dual' to $C$ ), will be called a partition of $R$ and denoted by $\mathcal{P}$. Given $\mathcal{P}$, for every $C \in \mathcal{C}$ and $s=\left(S_{1}, S_{2}, S_{3}\right)$ we define a functional on the classical phase space

$$
q_{C}[E]:=\frac{1}{3 !} \epsilon^{i j k} \eta_{a b c} E_{S_{a}}^{i} E_{S_{b}}^{j} E_{S_{c}}^{k},
$$

where, as before, $E_{S}^{i}=\frac{1}{2} \int_{S} e_{a b}^{i} d x^{a} \wedge d x^{b}$ is the ' $S$-smeared triad'. Clearly, $\left|q_{C}(E)\right| / L_{C}^{6}$, where $L_{C}$ is the (coordinate) size of $C$, approximates the determinant $q$ of the metric $q_{a b}$ (defined by the triad $E_{i}^{a}$ ) at any internal point of the cell-cube $C$. This approximate expression of $q$ naturally provides an approximate expression $V_{R}^{\mathcal{P}}[E]$ of the volume of the region $R$, associated with the partition $\mathcal{P}$

$$
V_{R}^{\mathcal{P}}[E]:=\sum_{C \in \mathcal{C}} \sqrt{\left|q_{C}[E]\right|} .
$$

Indeed, if we assume that, for some $\epsilon>0 L_{C}$ is bounded from above by $\epsilon$ (i.e., $L_{C}<\epsilon$ for every cell) and for each $\epsilon$ we fix a partition $\mathcal{P}_{\epsilon}$ as above, then for every triad $E$ we have:

$$
V_{R}^{\mathcal{P}_{\epsilon}}[E] \rightarrow V_{R}(E) \text { as } \epsilon \rightarrow 0 .
$$

Thus, in the classical theory, the phase space function $V_{R}(E)$ can be expressed in terms of the two-dimensionally smeared triads. Since we already have the quantum operators corresponding to smeared triads, in the next sub-section we will be able to construct regulated quantum operators $\hat{q}_{C}$. The removal of regulators will however be much more subtle. In particular, we will have to introduce a certain 'averaging' procedure. We will conclude this sub-section by justifying this procedure from a classical perspective.

Let $\mathcal{S}$ denote an $n$-parameter family of coordinate systems, containing and smoothly related to $x^{a}$. (As we will see in Section 4.1, in quantum theory one is led to a specific $\mathcal{S}$. In the classical theory, however,we can keep $\mathcal{S}$ general.) Let us label the points of $\mathcal{S}$ by $n$ parameters, say $\theta^{A}$. Then, repeating the steps given above, we obtain $n$-parameter families of regulated functionals of triads, $q_{C}^{\theta}[E]$ and $V_{R}^{\mathcal{P}_{\epsilon}(\theta)}[E]$. For each $\theta$, we obtain $V_{R}^{\mathcal{P}_{\epsilon}(\theta)}[E]$ such that $V_{R}^{\mathcal{P}_{\epsilon}(\theta)}[E] \rightarrow V_{R}(E)$ as $\epsilon \rightarrow 0$. Let us assume that the family of coordinate systems is such that the convergence is uniform in $\theta$. Then, 
we can introduce a (rather trivial) averaging procedure as follows. Given a normalized function $\mu(\theta)$ on $\mathcal{S}$ (i.e., $\int_{\mathcal{S}} d^{n} \theta \mu(\theta)=1$ ), set

$$
\begin{aligned}
q_{C}^{\mathrm{av}}[E] & :=\int_{\mathcal{S}} d^{n}(\theta) \mu(\theta) q_{C}^{\theta} \\
V_{R}^{\mathrm{av}}[E] & :=\sum_{C \in \mathcal{C}} \sqrt{\left|q_{C}^{\mathrm{av}}[E]\right|} .
\end{aligned}
$$

Then $V_{R}^{\text {av }}[E] \rightarrow V_{R}(E)$ as $\epsilon \rightarrow 0$. Like all other steps in the regularization procedure, averaging is of course unnecessary in the classical theory. However, we will see in Section 4 that it plays an important role in the quantum theory.

This regularization is called 'internal' because the regulated volume functional is expressed in terms of triads which are smeared over two surfaces passing through the interior of cells (see the condition $i$ ) in Section 3.3). As a result, the final operator will turn out to be sensitive to the relation between tangent vectors to the edges at vertices of graphs, i.e. to the intrinsic structure of the graph at vertices. In the Appendix, we discuss an 'external' regularization where the regulated volume functional is expressed in terms of triads smeared on the boundary of cells. This expression does not depend on the details of what happens inside any cell, whence the resulting quantum operator is sensitive only to the extrinsic structure which can be registered on the boundaries of cells surrounding vertices.

\subsection{Regularized Quantum Operators}

The regulated volume $V_{R}^{\mathcal{P}}$ of (3.4) depends on the classical phase space variables only through (two-dimensionally) smeared triads. Since we already have the quantum analogs $\hat{E}_{S}^{i}$ of these (see (2.9)), it is straightforward to define the regulated volume operator. The operator $\hat{q}_{C}$ corresponding to (3.3) is given simply by:

$$
\begin{aligned}
\hat{q}_{C}= & \frac{1}{3 !} \epsilon_{i j k} \eta_{a b c} \hat{E}_{S_{a}}^{i} \hat{E}_{S_{b}}^{j} \hat{E}_{S_{c}}^{k} \\
= & \frac{1}{48} \epsilon_{i j k} \eta_{a b c} \\
& \cdot \sum_{x_{1} \in S_{a}} \sum_{x_{2} \in S_{b}} \sum_{x_{3} \in S_{c}} \sum_{\left[e_{1}\right],\left[e_{2}\right],\left[e_{3}\right]} \kappa^{a}\left(\left[e_{1}\right]\right) \kappa^{b}\left(\left[e_{2}\right]\right) \kappa^{c}\left(\left[e_{3}\right]\right) J_{x_{1}, e_{1}}^{i} J_{x_{2}, e_{2}}^{j} J_{x_{3}, e_{3}}^{k},
\end{aligned}
$$

where we denoted

$$
\kappa^{d}([e]):=\kappa_{S_{d}}([e])
$$


and where $\left[e_{r}\right]$ runs through the set of germs starting at $x_{r}, r=1,2,3$. As in Section 2, although infinite sums are involved, the action of this operator on cylindrical functions is well-defined because the result has only a finite number of non-zero terms. To define the regulated volume operator $\hat{V}_{R}^{(\mathcal{P})}$, we need to take the absolute value and square-root of $\hat{q}_{C}$. For this, it is necessary to show that $\hat{q}_{C}$ is a self-adjoint operator. Now, we know that each $J_{x, e}^{i}$ is an essentially self-adjoint operator. Furthermore, whenever $\left[e_{1}\right]=\left[e_{2}\right]$, we have $\eta_{a b c} \kappa^{a}\left(\left[e_{1}\right]\right) \kappa^{b}\left(\left[e_{2}\right]\right) \kappa^{c}\left(\left[e_{3}\right]\right)=0$. Therefore, the products on the right side of (3.6) contain operators associated with distinct edges. These operators commute. Hence, the sum contains only products of commuting essentially self-adjoint operators. It is easy to verify that the right side of (3.6) is therefore an essentially self-adjoint operator on the domain $\mathrm{Cyl}^{(3)}$ of $C^{3}$ cylindrical functions. Hence, we can take its self-adjoint extension and a well-defined regulated volume operator via:

$$
\hat{V}_{R}^{\mathcal{P}}:=\sum_{C}\left|\hat{q}_{C}\right|^{\frac{1}{2}}
$$

By construction, $\hat{V}_{R}^{\mathcal{P}}$ is a non-negative self-adjoint operator. This is the quantum analog of the approximate volume functional $V_{R}^{\mathcal{P}}$. It depends on our choice of partition $\mathcal{P}$ of the region $R$.

\subsection{Removing the Regulator}

In the classical theory, it is straightforward to remove the regulator. We can begin with any partition $\mathcal{P}$ and let the cells $C$ shrink in any smooth fashion; in the limit $\epsilon \rightarrow 0$, we have $V_{R}^{\mathcal{P}_{\epsilon}}(E) \rightarrow V_{R}(E)$. In the quantum theory, on the other hand, the limiting procedure involves certain subtleties. More precisely, now one has to 'streamline' the limiting procedure by specifying appropriate restrictions on how the partition $\mathcal{P}$ is to be refined as $\epsilon$ tends to zero. Note however that such subtleties are a commonplace in quantum field theory. For example, in interacting scalar field theories in low dimensions one generally has to remove the regulators in a specific order and/or take limits keeping certain ratios of cut-offs and parameters of the theory constant. Similarly, in gauge theories based on lattices, to compute expectation values of Wilson loops in the continuum limit, one only allows rectangular lattices and the refinement of these lattices is often tailored to the Wilson loop in question. Indeed, some of these strategies seem so 'natural' that the restrictions involved often go unmentioned.

To remove the regulator in the quantum theory, we will proceed as follows. First, we will fix a graph $\gamma$ and consider the subspace $\mathrm{Cyl}_{R(\gamma)}$ of Cyl consisting of all states $\Psi_{\gamma^{\prime}}$ which are cylindrical with respect to a graph $\gamma^{\prime}$ whose range coincides with the range of $\gamma$. The regulated operators $\hat{q}_{C}$ and 
$\hat{V}_{R}^{\mathcal{P}}$ leave this subspace invariant. Hence, we can meaningfully focus just on $\mathrm{Cyl}_{R(\gamma)}$ and specify how the regulator is to be removed to obtain the operator $\hat{V}_{R}^{\gamma}$ on $\mathrm{Cyl}_{R(\gamma)}$ from $V_{R}^{\mathcal{P}}$. For that we will use $\gamma$. However, we will see that the operators will stay unchanged if we choose another graph $\gamma^{\prime}$ which has the same range as $\gamma$. Moreover, the operators will in fact preserve the space $\mathrm{Cyl}_{\gamma}$ of the cylindrical functions based on $\gamma$. By varying $\gamma$, we will thus obtain a family of operators on various $\mathrm{Cyl}_{\gamma}$. Finally, we will verify that they are compatible in the appropriate sense [7], i.e., together constitute a well-defined operator $\hat{V}_{R}$ on $\mathcal{H}$.

Let us then begin with the first step. Fix a graph $\gamma$ and focus on $\mathrm{Cyl}_{R(\gamma)}$. The allowed refinements of the partition of the region $R$ will depend on the graph $\gamma$. More precisely, we will assume that (for sufficiently small $\epsilon$ ) the permissible partitions $\mathcal{P}$ satisfy the following three conditions (see fig.2):

(i) every vertex of the graph $\gamma$ (within $R$ ) is contained in the interior of one of the cells, say $C$, and coincides with the intersection point of the triplet of two-surfaces $S_{1}, S_{2}, S_{3}$ assigned to $C$ by the partition $\mathcal{P}$;

(ii) if a cell $C$ does contain a vertex, say $v$, then $v$ is the unique isolated intersection point between the union of the three two-surfaces $S_{a}$ associated to $C$ and the range of $\gamma$; and,

(iii) if a cell $C$ does not contain any of the vertices, then the triplet of surfaces $S_{a}$ associated by $\mathcal{P}$ to $C$ intersects $\gamma$ at most at two points.

These requirements are quite easy to meet. Given any partition $\mathcal{P}$ in which the vertices of the graph $\gamma$ do not lie on the walls of the cells, the first condition can be met simply by choosing the surfaces $S_{a}$ appropriately (within cells containing vertices). Given a partition satisfying the first condition, the second and third conditions can be generically ${ }^{5}$ satisfied by a permissible refinement of that partition. Furthermore, once a refinement satisfying these two conditions is achieved, subsequent refinements needed in the limiting procedure automatically satisfy them. Nonetheless, these conditions do restrict the allowed partitions. As we will see, they ensure that the limiting operator is well-defined; if refinements are taken arbitrarily, in general the limit fails to exist.

Let us now suppose that the partition $\mathcal{P}$ satisfies these conditions and evaluate the action of the operator $\hat{q}_{C}$ on an element $\Psi_{\gamma^{\prime}}$ in $\mathrm{Cyl}_{R(\gamma)}$. Note that because of the first condition on the partition, a cell $C$ either contains

\footnotetext{
${ }^{5}$ The fact that the edges are not allowed to lie in the surfaces $S_{a}$ in any cell does impose a mild restriction on the permissible coordinate systems used to construct the partition $\mathcal{P}$. However, this restriction is imposed only for simplicity of presentation. Because of the averaging procedure of Section 4, contributions from such non-generic partitions to the final result are negligible.
} 


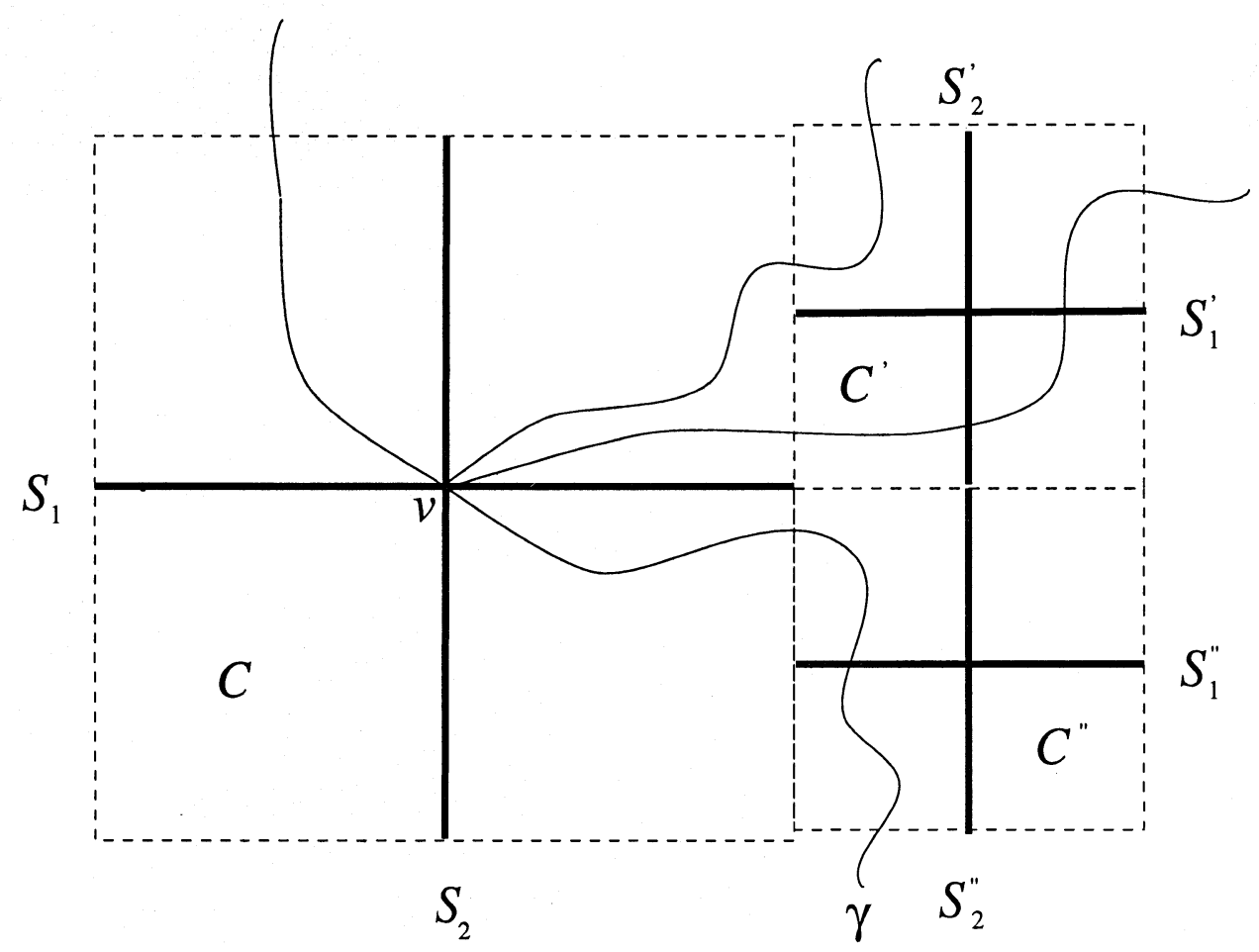

Figure 2: The figure illustrates the way cells $C, C^{\prime}, C^{\prime \prime}$ (the dashed lines) and 2-surfaces $S_{a}, S^{\prime}{ }_{a}, S^{\prime \prime}{ }_{a}$ (the bold faced lines) of a partition $\mathcal{P}$ are adapted to a neighborhood of a vertex $v$ of a graph $\gamma$ according to the conditions $i$ )-iii) above. For simplicity, one dimension has been dropped.

one vertex of $\gamma$ or no vertex at all. If it does not contain a vertex, then by condition (iii) on the partition, due to anti-symmetrization forced by $\eta_{a b c}$, (3.6) reduces to

$$
\hat{q}_{C} \Psi_{\gamma}=0 .
$$

If the cell $C$ does contain a vertex, say $v$, then (3.6) and condition (ii) on the partition implies:

$$
\hat{q}_{C} \Psi_{\gamma}=\frac{1}{48} \sum_{I, J, K} \epsilon_{i j k} \epsilon_{a b c} \kappa^{a}\left(\left[e_{I}\right]\right) \kappa^{b}\left(\left[e_{J}\right]\right) \kappa^{c}\left(\left[e_{K}\right]\right) J_{v, e_{I}}^{i} J_{v, e_{J}}^{j} J_{v, e_{K}}^{k} \Psi_{\gamma},
$$

where $I, J, K$ label the edges of $\gamma$ passing through the vertex $v$. For simplicity, we will refer to $\kappa^{a}\left[e_{I}\right]$ as 'vectors'. The possible 'components' of these vectors are $0, \pm 1$ and depend only on the octants defined by the two-surfaces $\left(S_{1}, S_{2}, S_{3}\right)$ which contains the edge $e_{I}$. To summarize, the conditions on permissible partitions have streamlined the calculation by reducing (3.6) to (3.9) and (3.10). 
Let us now focus on the non-trivial case in which $C$ contains a vertex $v$. The action of the operator $\hat{q}_{C}$ depends on the three two-surfaces $S_{a}$ only through the properties of these surfaces at $v$. Hence, it is unchanged as we refine the partition and shrink the cell $C$ to $v$ :

$$
\lim _{\epsilon \rightarrow 0} \hat{q}_{C} \Psi_{\gamma}=\frac{1}{48} \sum_{I, J, K} \kappa\left(\left[e_{I}\right],\left[e_{J}\right],\left[e_{K}\right]\right) \epsilon_{i j k} J_{v, e_{I}}^{i} J_{v, e_{J}}^{j} J_{v, e_{K}}^{k} \Psi_{\gamma}
$$

where the sum is over the edges passing through $v$ and where

$$
\kappa\left(\left[e_{I}\right],\left[e_{J}\right],\left[e_{K}\right]\right):=\epsilon_{a b c} \kappa^{a}\left(\left[e_{I}\right]\right) \kappa^{b}\left(\left[e_{J}\right]\right) \kappa^{c}\left(\left[e_{K}\right]\right)
$$

(In particular, $\kappa\left(\left[e_{I}\right],\left[e_{J}\right],\left[e_{K}\right]\right)$ vanishes if any two of the edges $e_{I}, e_{J}, e_{K}$ lie in the same or opposite octants.) Using the arguments used in the last sub-section, one can show that the limiting operator is well-defined and essentially self-adjoint on the Hilbert space $H_{\gamma}$ obtained by Cauchy completing $\mathrm{Cyl}_{\gamma}$. Now, if given the space $\mathrm{Cyl}_{R(\gamma)}$ we used in the above construction a different graph $\gamma^{\prime}$ whose range is the same as that of $\gamma$, the only difference is that $\gamma^{\prime}$ may have some extra bivalent vertices and/or some bivalent vertices of $\gamma$ may be missing in the set of vertices of $\gamma^{\prime}$. However, for a bivalent vertex, the operator $\hat{q}_{C}$ vanishes identically. Therefore the resulting volume operator derived in $\mathrm{Cyl}_{R(\gamma)}$ using $\gamma^{\prime}$ coincides with that for $\gamma$.

Unfortunately, however, this limiting operator (3.11) carries a memory of our choice of partitions through the term $\kappa\left(\left[e_{I}\right],\left[e_{J}\right],\left[e_{K}\right]\right)$, i.e., on the background structure used in the regularization procedure. Hence, although the limit of the operator $\hat{V}_{R}^{(\mathcal{P})}$ of $(3.8)$ is well-defined, it does not lead to a viable candidate for the volume operator. However, since the background dependence is of a rather simple type, one can eliminate it by suitably 'averaging' the regularized operator over relevant background structures. We will carry out this averaging in the next section.

To conclude this sub-section, let us note two properties of the limiting operator (3.11) which follow by inspection. Irrespective of the choice of permissible partitions used in the regularization procedure, we have:

(1) $\kappa\left(\left[e_{I}\right],\left[e_{J}\right],\left[e_{K}\right]\right)$ depends only on the germs of the edges; and,

(2) $\kappa\left(\left[e_{I}\right],\left[e_{J}\right],\left[e_{K}\right]\right)$ is totally anti-symmetric in its three arguments. In particular therefore, if a graph has only bivalent ${ }^{6}$ vertices, the limit of the regularized operator $\hat{q}_{C}$ annihilates all states in $\mathrm{Cyl}_{\gamma}$.

We will see that these properties are trivially preserved by averaging and are therefore shared by the final volume operator $\hat{V}_{R}$ we will obtain in the next section.

\footnotetext{
${ }^{6}$ If $\gamma$ has (at most) trivalent vertices, simple algebra shows that the action of the limiting operator on all gauge invariant states in $\mathrm{Cyl}_{\gamma}$ also vanishes [21]. See Section 5.
} 


\section{Averaging}

To remove the background dependence, we need to appropriately average $\hat{q}_{C}$ over the relevant background structures, use the resulting operator $\hat{q}_{C}^{\text {av }}$ in place of $\hat{q}_{C}$ in $(3.8,3.6)$, and then take the limit. Our discussion is divided into four parts. In the first, we spell out the basic strategy. In the second, we show that the desired symmetries of the final volume operator and the consistency requirement on the regularization procedure fix the form of the averaged quantity $\kappa^{\mathrm{av}}\left(\left[e_{I}\right],\left[e_{J}\right],\left[e_{K}\right]\right)$ - and hence also of the final volume operatoruniquely up to a multiplicative constant. In the third, we establish the existence of certain measures that are needed for the averaging procedure. Finally, in the forth part, we collect results of the Sections 3 and $4.1-4.3$ to arrive at the desired volume operator.

\subsection{Basic Strategy}

The background dependence in the limit of the regularized volume operator $(3.8,3.11)$ appears only through the factors $\kappa\left(\left[e_{I}\right],\left[e_{J}\right],\left[e_{K}\right]\right)$ associated with cells containing a vertex of $\gamma$. Therefore, let us first focus only on a single cell $C$ containing a vertex $v$. Note that $\kappa\left(\left[e_{I}\right],\left[e_{J}\right],\left[e_{K}\right]\right)$ depends only on the relation between the three edges and the coordinate octants at the vertex $v$. Therefore, while there is an infinite-dimensional freedom in the choice of the background coordinates with which we began, the 'relevant' freedom for averaging turns out to be only finite-dimensional. To see this, let us regard two coordinate systems in $C$, centered at $v$, as equivalent if they yield the same $\kappa\left(\left[e_{I}\right],\left[e_{J}\right],\left[e_{K}\right]\right)$ for all triplets of edges of $\gamma$ passing through $v$. Then, given two equivalence classes and a coordinate system belonging to the first, one can generically ${ }^{7}$ find a system in the second which is related to the first by the action of $G L^{+}(3)$, the group of orientationpreserving general linear transformations at $v$. Furthermore, the diagonal subgroup diag of $G L^{+}(3)$ merely rescales the coordinates and hence leaves each $\kappa^{a}\left[e_{I}\right]$ unchanged. Hence, to get rid of the background dependence, it suffices to average $\kappa\left(\left[e_{I}\right],\left[e_{J}\right],\left[e_{K}\right]\right)$ only on the finite-dimensional coset-space $G L^{+}(3) /$ diag. Topologically, this space $\mathcal{S}$ of 'relevant' background structures can be identified with an open subset of $S^{2} \times S^{2} \times S^{2}$ and coordinatized by six angular coordinates, $\theta^{A}$ say, with $A=1, \ldots, 6$.

Our task is to average the operator $\hat{q}_{C}$ over the space $\mathcal{S}$ in such a way that the resulting volume operator $\hat{V}_{R}$ is well-defined and covariant, i.e., has no memory of the background structures used in the regularization procedure. Fix a coordinate system $x^{a}$ in an open region $U$ within $\Sigma$ containing our region $R$ and an adapted, permissible partition $\mathcal{P}$ of $R$ as in Section 3.3. Fix

\footnotetext{
${ }^{7} \mathrm{We}$ can focus on the generic case because the averaging procedure involves integration and lets us ignore 'sets of measure zero'.
} 
a vertex $v$ of $\gamma$ and, as before, denote by $C$ the cell containing $v$. Given a $\theta^{A} \in \mathcal{S}$, we obtain a second coordinate system $x^{a}(\theta)$, obtained from the first by the action of an element of $G L^{+}(3)$ corresponding to $\theta^{A}$ (around some fixed origin in $R$ ). Using this coordinate system, we can again construct a permissible partition $\mathcal{P}(\theta)$ of $R$. Denote by $C(\theta)$ the cell in this partition containing the vertex $v$. Replacing $C$ by $C(\theta)$ in Section 3.3, for each $\theta \in \mathcal{S}$, we obtain an operator $\hat{q}_{C}^{\theta}$ on $\mathrm{Cyl}_{\gamma}^{(1)}$. We wish to average these operators with respect to a suitable probability measure on $\mathcal{S}$. Now, given any normalized function $\mu(\theta)$, (i.e. satisfying $\int_{\mathcal{S}} d^{6} \theta \mu(\theta)=1$ ) the average $\hat{q}_{C}^{\text {av }}$ of $\hat{q}_{C}^{\theta}$ is given by:

$$
\begin{aligned}
\hat{q}_{C}^{\mathrm{av}} \Psi_{\gamma} & =\int_{\mathcal{S}} d^{6} \theta \mu(\theta) \hat{q}_{C}^{\theta} \Psi_{\gamma} \\
& =\frac{1}{48} \sum_{I, J, K} \kappa^{\mathrm{av}}\left(\left[e_{I}\right],\left[e_{J}\right],\left[e_{K}\right]\right) \epsilon_{i j k} J_{v, e_{I}}^{i} J_{v, e_{J}}^{j} J_{v, e_{K}}^{k} \Psi_{\gamma}
\end{aligned}
$$

where

$$
\kappa^{\mathrm{av}}\left(\left[e_{I}\right],\left[e_{j}\right],\left[e_{K}\right]\right)=\int_{\mathcal{S}} d^{6} \theta \mu(\theta) \kappa\left(\left[e_{I}\right],\left[e_{J}\right],\left[e_{K}\right], \theta\right)
$$

Thus, for any normalized measure $\mu$, the averaged operators $\hat{q}_{C}^{\text {av }}$ are welldefined. Using these in place of $\hat{q}_{C}$ in (3.8), one can construct the regularized volume operator $\hat{V}_{R}^{\text {av }}$. As in Section 3 , it is straightforward to remove the regulator. So far, for simplicity of presentation, we have focussed on a single cell $C$ containing a vertex $v$. However, since the coordinate systems $x^{a}(\theta)$ were not adapted to any specific cell, (4.1) and (4.2) hold for all cells.

To summarize, the basic idea is simply to repeat the procedure of section 3 but now using averaged operators. Physical justification for this strategy comes from the fact that, as we saw in Section 3.1, the averaging procedure does yield the correct volume functional in the classical theory.

The key question then is: what measure should we use for averaging? Unfortunately, the space $\mathcal{S}$ is non-compact and does not admit a canonical normalized measure. Can a suitable measure be perhaps selected by examining the classical limit? The answer is in the negative: As we saw in Section 3.1 , although the averaging procedure is applicable classically, the averaged regulated volume tends to $V_{R}$ for any normalized $\mu(\theta)$. In the quantum theory, on the other hand, this is not the case and we need to find $\mu(\theta)$ for which the final volume operator is background independent. In Section 4.2 we will assume that such measures exist and show that the requirement of covariance of the final volume operator determines $\kappa^{\text {av }}$ - and hence also the final volume operator - uniquely up to a multiplicative constant. Existence of measures of the required type will then be established in Section 4.3. 


\subsection{Uniqueness of $\kappa^{\mathrm{av}}$}

Let us suppose that there does exist a normalized measure $\mu$ on $\mathcal{S}$ such that the resulting volume operator $\hat{V}_{R}$ transforms covariantly under diffeomorphisms of $\Sigma$. What can we say about the corresponding $\kappa^{\text {av }}\left(\left[e_{I}\right],\left[e_{J}\right],\left[e_{K}\right]\right)$ ? The purpose of this sub-section is to show that, given any cell containing a vertex $v$ of any graph, the quantity must be of the type:

$$
\kappa^{\mathrm{av}}\left(\left[e_{I}\right],\left[e_{J}\right],\left[e_{K}\right]\right)=\kappa_{(\mu)} \epsilon\left(e_{I}, e_{J}, e_{K}\right)
$$

for some (measure-dependent) constant $\kappa_{(\mu)}$, where $\epsilon\left(e_{I}, e_{J}, e_{K}\right)$ is the orientation function which equals 0 if the tangent directions ${ }^{8}$ to the three edges are linearly dependent at the vertex $v$, and \pm 1 if they are linearly independent and oriented positively or negatively. (Recall that $\Sigma$ is oriented.) Thus, the measure dependence is contained in a single, overall multiplicative constant.

The idea is to use symmetries of $\kappa^{\mathrm{av}}\left(\left[e_{I}\right],\left[e_{J}\right],\left[e_{K}\right]\right)$ implied by (3.12), (4.2), and the requirement that $\hat{V}_{R}$ be diffeomorphism covariant, to constrain its form. We saw at the end of the last sub-section that $\kappa\left(\left[e_{I}\right],\left[e_{J}\right],\left[e_{K}\right]\right)$ has two properties irrespective of the choice of a permissible partition. It is trivial to verify that these properties are preserved by averaging. Thus, we have:

(1) $\kappa^{\mathrm{av}}\left(\left[e_{I}\right],\left[e_{J}\right],\left[e_{K}\right]\right)$ depends only on the germs of the edges; and,

(2) $\kappa^{\mathrm{av}}\left(\left[e_{I}\right],\left[e_{J}\right],\left[e_{K}\right]\right)$ is totally anti-symmetric in its three arguments.

Next, recall that a cylindrical function in $\mathrm{Cyl}_{\gamma}$ is also cylindrical with respect to a graph $\gamma^{\prime}$ with $\gamma^{\prime} \geq \gamma$, i.e., such that (the range of) $\gamma$ is contained in (the range of) $\gamma^{\prime}$. Since we want the volume operator $\hat{V}_{R}$ to be well-defined at the end, its action on a state should not depend on whether we regard the state as being cylindrical with respect to the first graph or the second. This implies that

(3) the function $\left(e_{I}, e_{J}, e_{K}\right) \rightarrow \kappa^{\mathrm{av}}\left(\left[e_{I}\right],\left[e_{J}\right],\left[e_{K}\right]\right)$ defined by $\kappa^{\mathrm{av}}\left(\left[e_{I}\right]\right.$, $\left.\left[e_{J}\right],\left[e_{K}\right]\right)$ depends only on the germs of the three edges and not on the specific graph used in the computation. Thus, the averaging procedure simply provides a function from germs of (ordered) triplets of edges intersecting at any point $x$ in $\Sigma$ to the reals.

Next, the assumed diffeomorphism covariance of the volume operator implies that this function must have the following property:

\footnotetext{
${ }^{8} \mathrm{~A}$ germ $[e]$ at a point $x$ in $\Sigma$ defines a unique oriented tangent direction at $x$.
} 
(4) Given any two triplets $\left(e_{I}, e_{J}, e_{K}\right)$ and $\left(e_{I}^{\prime}, e_{J}^{\prime}, e_{K}^{\prime}\right)$ of edges related by an orientation-preserving diffeomorphism of $\Sigma$,

$$
\kappa^{\mathrm{av}}\left(\left[e_{I}\right],\left[e_{J}\right],\left[e_{K}\right]\right)=\kappa^{\mathrm{av}}\left(\left[e_{I}^{\prime}\right],\left[e_{J}^{\prime}\right],\left[e_{K}^{\prime}\right]\right) .
$$

The next property follows immediately from (2)-(4).

(5) Let the triplet be such that the tangent directions they define at $x$ are linearly independent. Then,

$$
\kappa^{\mathrm{av}}\left(\left[e_{I}\right],\left[e_{J}\right],\left[e_{K}\right]\right)=\kappa_{(\mu)} \epsilon\left(e_{I}, e_{J}, e_{K}\right)
$$

since this is the only diffeomorphism invariant, totally anti-symmetric function of germs of ordered triplets of edges intersecting at $x$.

On the other hand, if the three tangent directions are linearly dependent, other invariants exist, which depend on higher derivatives of edges at the intersection point. A priori, these are potential candidates for $\kappa^{\mathrm{av}}\left(\left[e_{I}\right]\right.$, $\left.\left[e_{J}\right],\left[e_{K}\right]\right)$. However, they can be ruled out as follows. Consider two triplets with the same orientation, $\left(\left[e_{1}\right],\left[e_{2}\right],\left[e_{3}\right]\right)$ and $\left(\left[e_{1}\right],\left[e_{2}\right],\left[e_{3}^{\prime}\right]\right)$, such that $e_{3}^{\prime}$ is tangent to $e_{3}$ at $x$. For a point $\theta \in \mathcal{S}$ such that none of the corresponding two-surfaces $S_{a}$ passing through $v$ is tangent to any of the germs $e_{1}, e_{2}, e_{3}$, the germs $e_{3}$ and $e_{3}^{\prime}$ are on the same side of each of the two-surfaces. Therefore, almost everywhere on $\mathcal{S}$, we have $\kappa_{S_{a}}\left(e_{3}\right)=\kappa_{S_{a}}\left(e^{\prime}{ }_{3}\right)$. Hence, whenever the three pairs of primed and unprimed germs define the same oriented tangent directions at $x$, the equality

$$
\kappa\left(\left[e_{I}\right],\left[e_{J}\right],\left[e_{K}\right], \theta\right)=\kappa\left(\left[e_{I}^{\prime}\right],\left[e_{J}^{\prime}\right],\left[e_{K}^{\prime}\right], \theta\right)
$$

holds almost everywhere on $\mathcal{S}$. In this case, integration with respect to a $\mu(\theta) d^{6} \theta$ yields

$$
\kappa^{\mathrm{av}}\left(\left[e_{I}\right],\left[e_{J}\right],\left[e_{K}\right]\right)=\kappa^{\mathrm{av}}\left(\left[e_{I}^{\prime}\right],\left[e_{J}^{\prime}\right],\left[e_{K}^{\prime}\right]\right) .
$$

To summarize, we have shown that

(6) given a point $x$ in $\Sigma, \kappa^{\mathrm{av}}\left(\left[e_{I}\right],\left[e_{J}\right],\left[e_{K}\right]\right)$ can depend only on the oriented tangent directions at $x$ defined by the three germs.

We can now use this property to evaluate $\kappa^{\text {av }}\left(\left[e_{I}\right],\left[e_{J}\right],\left[e_{K}\right]\right)$ in the case when the tangent directions are linearly dependent. If two of these directions coincide, anti-symmetry immediately implies that $\kappa^{\mathrm{av}}\left(\left[e_{I}\right],\left[e_{J}\right],\left[e_{K}\right]\right)$ must vanish. Next, note that (2.8) implies that $\kappa_{S_{a}}\left[e_{I}\right]=-\kappa_{S_{a}}\left[-e_{I}\right]$. Hence, $\kappa^{\mathrm{av}}\left(\left[e_{I}\right],\left[e_{J}\right],\left[e_{K}\right]\right)=-\kappa^{\mathrm{av}}\left(\left[-e_{I}\right],\left[e_{J}\right],\left[e_{K}\right]\right)$, which in turn implies that $\kappa^{\text {av }}\left(\left[e_{I}\right],\left[e_{J}\right],\left[e_{K}\right]\right)$ vanishes if any two tangent directions are anti-parallel. Finally, consider the remaining case in which the three tangent directions 
span a two-plane but are such that no two of these directions are tangential to each other. Then, modulo a possible diffeomorphism (to 'straighten out the edges'), there is a coordinate system in $\mathcal{S}$ in which (the point $x$ lies at the origin and) the three germs coincide with straight lines $(1,0,0),(0,1,0)$ and $(1,1,0)$. Finally, there exists an orientation-preserving diffeomorphism -the rotation through $\pi$ along the third axis- which carries $\left(\left[e_{1}\right],\left[e_{2}\right],\left[e_{3}\right]\right)$ to $\left(\left[e_{2}\right],\left[e_{1}\right],\left[e_{3}\right]\right)$. Hence, by diffeomorphism invariance and anti-symmetry, we conclude $\kappa^{\mathrm{av}}\left(\left[e_{I}\right],\left[e_{J}\right],\left[e_{K}\right]\right)=0$ in this case as well. Thus, we have:

(7) If the three tangent directions determined by the edges $\left(\left[e_{I}\right],\left[e_{J}\right],\left[e_{K}\right]\right)$ at $x$ are linearly dependent, then $\kappa^{\mathrm{av}}\left(\left[e_{I}\right],\left[e_{J}\right],\left[e_{K}\right]\right)=0$.

Properties (5) and (7) imply that if there exists a measure $\mu$ on $\mathcal{S}$, averaging with respect to which provides a well-defined diffeomorphism covariant volume operator $\hat{V}_{R}$, then that averaging determines $\kappa^{\mathrm{av}}\left(\left[e_{I}\right],\left[e_{J}\right],\left[e_{K}\right]\right)$ uniquely, up to a multiplicative constant $\kappa_{(\mu)}$. It is given by (4.3). Finally, properties (3) and (6) imply that the constant $\kappa_{(\mu)}$ can depend only on the averaging function $\mu(\theta)$ and not on the specific vertex or graph under consideration.

\subsection{Existence of Required Measures}

We now turn to the issue of existence: do there exist averaging functions $\mu(\theta)$ which lead to volume operators $\hat{V}_{R}$ that transform covariantly under diffeomorphisms? We just saw in Section 4.2 that the necessary and sufficient condition for $\hat{V}_{R}$ to be well-defined and covariant is that $\kappa^{\text {av }}$ of (4.2) be given by (4.5). Therefore, we can rephrase the question as follows: Given any vertex $v$ of any graph $\gamma$, does there exist a function $\mu(\theta)$ on $\mathcal{S}$ such that, for any triplet $e_{I}, e_{J}, e_{K}$ of edges of $\gamma$ at the vertex,

$$
\int_{\mathcal{S}} d^{6} \theta \mu(\theta) \kappa\left(\left[e_{I}\right],\left[e_{J}\right],\left[e_{K}\right], \theta\right)=\kappa_{o} \epsilon\left(\left[e_{1}\right],\left[e_{2}\right],\left[e_{3}\right]\right) \quad \text { and } \quad \int_{\mathcal{S}} d^{6} \theta \mu(\theta)=1
$$

for some constant $\kappa_{o}$ ?

Now, since $\kappa\left(\left[e_{I}\right],\left[e_{J}\right],\left[e_{K}\right]\right)$ depends only on the oriented tangent directions of the three edges and since the integrals in the above equations represent the $L^{2}$ inner product on $\left(\mathcal{S}, d^{6} \theta\right)$ (of $\mu$ with $\kappa$ and 1 respectively) the required $\mu(\theta)$ is guaranteed to exist provided the following statement holds: For any finite set of germs $\left\{\left[e_{1}\right], \ldots,\left[e_{N}\right]\right\}$ at a point $v$, such that $e_{I}$ is not tangent to $e_{J}$ whenever $I \neq J$, the functions $\kappa\left(\left[e_{I}\right],\left[e_{J}\right],\left[e_{K}\right], \theta\right)$, $I<J<K$ and the constant function constitute a set of linearly independent functions on $\mathcal{S}$.

Let us therefore establish this statement. Recall first that the set of regulators $\mathcal{S}$ can be identified with the set of triplets of oriented two-surfaces, 
$\theta \equiv\left(S_{a}^{\prime}\right)_{a=1,2,3}$, intersecting at $v$. (These triplets are obtained by applying the orientation preserving $G L(3)$ linear transformations on the surfaces $x^{a}=$ const, the action of $G L(3)$ being defined with respect to this initial coordinate system.) Now, given germs $\left[e_{1}\right], \ldots,\left[e_{N}\right]$ as in the statement, suppose that there exist constants $a, a_{I J K}, I, J, K=1, \ldots, N$ such that

$$
a+\sum_{I<J<K} a_{I J K} \kappa\left(\left[e_{I}\right],\left[e_{J}\right],\left[e_{K}\right], \theta\right)==0
$$

for almost every $\theta=\left(S_{1}, S_{2}, S_{3}\right) \in \mathcal{S}$. Pick a two-surface $S_{0}$ which is tangent to $\left[e_{1}\right]$ but is not to any of the other germs. Consider the points of $\mathcal{S}$ where $S_{1}$ is near $S_{0}$. Then, as we slightly vary $S_{1}$ around $S_{0}$ : the functions $\kappa_{S_{1}}\left(e_{J}\right)$ remain unchanged if $J \neq 1$. On the other hand, $\kappa_{S_{1}}\left(e_{1}\right)$ does change. We can find two-surfaces $S_{-}$and $S_{+}$near $S_{0}$ such that

$$
\kappa_{S_{ \pm}}\left(e_{I}\right)= \pm 1 \text {. }
$$

Therefore, plugging into (4.8) $\theta_{-}:=\left(S_{-}, S_{2}, S_{3}\right)$, and next $\theta_{+}=\left(S_{+}, S_{2}, S_{3}\right)$ with any $S_{2}, S_{3}$, and subtracting, we find

$$
a_{1 J K} \epsilon_{1 b c} \kappa_{S_{b}}\left(e_{J}\right) \kappa_{S_{c}}\left(e_{K}\right)=0,
$$

for arbitrary $S_{2}, S_{3}$. Repeating that argument for $e_{2}$ and $e_{3}$, say, we obtain

$$
a_{123}=0 \text {. }
$$

Since this argument applies to any triple $I, J, K$, we conclude

$$
a_{I J K}=0=a .
$$

Thus, as asserted in the statement above, the constiant function and the functions $\kappa\left(\left[e_{I}\right],\left[e_{J}\right],\left[e_{K}\right], \theta\right)$ are all linearly independent on $\mathcal{S}$.

This establishes the existence of averaging functions of the required type.

\subsection{Summary}

Let us now collect the results obtained in Section 3 and the previous three subsections. In Section 3, we began by recasting the volume function $V_{R}$ on the classical phase space such that the resulting 'regulated' form could be taken over to the quantum theory. We then showed that if the regulator is removed with due care, the limiting operator $(3.8,3.11)$ is well-defined. However, it carries a memory of the regulators used and therefore the resulting volume operator fails to be covariant under diffeomorphisms. To remedy this situation, in Section 4 we introduced a procedure to average over the 'relevant' background structures. It turns out that the requirement 
that the final operator $\hat{V}_{R}$ be well-defined and diffeomorphism covariant is so strong that it determines the form of the averaged operator except for an overall multiplicative constant $\kappa_{o}$. Finally, this form does result from averaging with respect to suitable measures, i.e., the averaging functions of the required type do exist.

The final result is the following. Given a region $R$ in $\Sigma$, we have an operator $\hat{V}_{R}$, whose action on any cylindrical function $\Psi_{\gamma}$ is given by

$$
\hat{V}_{R} \Psi_{\gamma}=\kappa_{o} \sum_{v} \sqrt{\left|\hat{q}_{v}\right|} \Psi_{\gamma}
$$

where

$$
\hat{q}_{v} \Psi_{\gamma}=\frac{1}{48} \epsilon_{i j k} \sum_{e, e^{\prime}, e^{\prime \prime}} \epsilon\left(e, e^{\prime}, e^{\prime \prime}\right) J_{v, e}^{i} J_{v, e^{\prime}}^{j} J_{v, e^{\prime \prime}}^{k} \Psi_{\gamma} .
$$

Here $v$ runs over the set of vertices of $\gamma ; e, e^{\prime}, e^{\prime \prime}$ over the set of edges of $\gamma$ passing through the vertex $v$ and $\epsilon\left(e, e^{\prime}, e^{\prime \prime}\right)$ is the orientation function, which equals 0 of the tangent directions of the three edges are linearly dependent and \pm 1 if they are linearly independent and oriented positively or negative with respect to the fixed orientation on $\Sigma$. The constant $\kappa_{o}$ remains undetermined. As written, because of the explicit reference to the graph $\gamma$, the operator is defined in $\mathrm{Cyl}_{\gamma}^{(3)}$ (for any $\gamma$ ). Next, it is quite easy to check that for any pair of graphs, the corresponding operators agree on the intersection $\mathrm{Cyl}_{\gamma}^{(3)} \cap \mathrm{Cyl}_{\gamma^{\prime}}^{(3)}$. That is, the family of operators defined on various $\mathrm{Cyl}_{\gamma}^{(3)}$ satisfy the 'cylindrical consistency' condition introduced in [7]). Therefore, (4.13) unambiguously defines an operator in $\mathrm{Cyl}^{(3)}$.

We conclude with two remarks.

1) Restoration of constants: Let us restore the factors of $c, G, \hbar$ and the Immirzi parameter $\gamma$ in the final expression. In the quantum representation labelled by $\gamma$, the volume operator is given by:

$$
\hat{V}_{R} \Psi_{\gamma}=\kappa_{o}\left(\frac{8 \pi G \hbar \gamma}{c^{3}}\right)^{\frac{3}{2}} \sum_{v} \sqrt{\left|\hat{q}_{v}\right|} \Psi_{\gamma}
$$

where

$$
\hat{q}_{v} \Psi_{\gamma}=\frac{1}{48} \epsilon_{i j k} \sum_{e, e^{\prime}, e^{\prime \prime}} \epsilon\left(e, e^{\prime}, e^{\prime \prime}\right) J_{v, e}^{i} J_{v, e^{\prime}}^{j} J_{v, e^{\prime \prime}}^{k} \Psi_{\gamma}
$$

In the remainder of the paper, however, we return to the conventions $c=1$, $8 \pi G=1, \hbar=1$ and $\gamma=1$.

2) Extension to the smooth case: As we remarked in footnote 2, the analyticity of the graphs is not really essential for the results presented above. 
We can begin with the vector space spanned by the set of cylindrical functions $\mathrm{Cyl}_{\infty}$ given by all the smooth graphs, replacing 'analytic' by 'smooth' in the definition of a graph, following [28]. The work of Baez and Sawin [5] provides a natural extension of the integral $\int d \mu_{o}$ clefined for elements of $\mathrm{Cyl}_{\infty}$. The Cauchy completion of this space then leads to a Hilbert space. In the regularization of volume, the only potential problem with this extension is that the domain of the operator $\hat{E}_{S}^{i}$ smeared over a two-surface $S$ (which may or may not be analytic) fails to be dense in the Hilbert space. This problem occurs because a smooth graph can intersect a given twosurface in an infinite number of isolated points. Therefore, to define the regulated operators $(3.8,3.6)$ we need to start with $c_{\iota}$ partition $\mathcal{P}$ that satisfies the conditions $(i)-($ iii $)$ of Section 3.3. Then, given a graph $\gamma$ the number of intersections between the two-surfaces $S_{a}$ defined by $\mathcal{P}$ and $\gamma$ is finite by construction. Hence, the two-surface operators $E_{S_{a}}^{i}$ are well defined in $\mathrm{Cyl}_{R(\gamma)}$ and the entire construction goes through. The resulting volume is given by the same formula (4.14) and has all the properties discussed below. One of them, the diffeomorphism invariance, is even easier to formulate because the action of the smooth diffeomorphisms is now well defined on the whole Hilbert space. (However, the arguments used should be modified: one has to use the spin-network decomposition from the beginning.) Using analogous modifications, the 'external' regularization of volume presented in the Appendix can also be extended to the smooth case.

\section{Properties of the Volume Operator}

Although the volume operators $\hat{V}_{R}$ are not as well understood as their area counterparts, their basic properties have been explored. The purpose of this section is to summarize these.

\subsection{Preliminaries}

1) As with area operators, the expression of $\hat{V}_{R}$ can be recast in an 'intrinsic' fashion that does not refer to any graphs.

$$
\hat{V}_{R}=\kappa_{o} \sum_{x \in R} \sqrt{\left|\hat{q}_{x}\right|}
$$

where

$$
\hat{q}_{x}=\frac{1}{48} \sum_{[e],\left[e^{\prime}\right],\left[e^{\prime \prime}\right]} \epsilon_{i j k} \epsilon\left(e, e^{\prime}, e^{\prime \prime}\right) J_{x, e}^{i} J_{x, e^{\prime}}^{j} J_{x, e^{\prime \prime}}^{k},
$$

where each of $[e],\left[e^{\prime}\right]$ and $\left[e^{\prime \prime}\right]$ runs through the set of germs of one-dimensional submanifolds of $\Sigma$, bounded from one side by $x$. As usual, 
acting on a cylindrical function $\Psi_{\gamma}$ the action is non-trivial only if $x$ is a vertex of the graph and the germs $[e],\left[e^{\prime}\right],\left[e^{\prime \prime}\right]$ overlap three edges of $\gamma$ intersecting at $x$. In the terminology of [7] the operator $\hat{q}_{x}$ is given by a cylindrically consistent family - labelled by all the graphs - of essentially self-adjoint operators $\mathrm{Cyl}_{\gamma}^{(3)} \rightarrow \mathrm{Cyl}_{\gamma}^{(0)}$. Hence, with domain $\mathrm{Cyl}^{(3)}$, it is an essentially self-adjoint operator on $\mathcal{H}$. Therefore the absolute value and square root of this operator used in the first equality of (5.1) are well defined and $\hat{V}_{R}$ is essentially self-adjoint.

2) In view of the above formulas it is meaningful to regard the operator $\widehat{\sqrt{q}(x)}$, representing the square root of the determinant of the metric, as an operator-valued distribution:

$$
\widehat{\sqrt{q(x)}} \Psi_{\gamma}=\frac{\kappa_{o}}{\sqrt{48}} \sum_{v} \delta^{3}(x, v)\left|\sum_{[e],\left[e^{\prime}\right],\left[e^{\prime \prime}\right]} \epsilon\left(e, e^{\prime}, e^{\prime \prime}\right) J_{x, e}^{i} J_{x, e^{\prime}}^{j} J_{x, e^{\prime \prime}}^{k}\right|^{\frac{1}{2}} \Psi_{\gamma} .
$$

By contrast, the determinant of the metric fails to exist even as an operatorvalued distribution. This is completely analogous to the situation for determinants of 2-metrics that was encountered in the discussion of area operators.

3 ) If $R(x, \epsilon)$ is a family of neighborhoods which shrink to $x$ as $\epsilon \rightarrow 0$, then given any $C^{3}$ cylindrical state $\Psi_{\gamma}$, the limit

$$
\lim _{\epsilon \rightarrow 0} \hat{V}_{R(x, \epsilon)} \Psi_{\gamma}
$$

exists but in general is not zero. This property plays an important role in the recent regularizations of various operators that arise in quantum dynamics $[14,27]$.

\subsection{The Gauge Invariance and Diffeomorphism Covariance.}

Both $\hat{V}_{R}$ and $\hat{q}_{x}$ are gauge invariant. Therefore, they naturally restrict to the operators in the space of gauge invariant cylindrical functions $\mathrm{Cyl}^{(3)}(\overline{\mathcal{A}} / \overline{\mathcal{G}})$.

Since the edges of our graphs are analytic, analytic diffeomorphisms on $\Sigma$ have a well-defined action on $\mathrm{Cyl}^{(3)}$. The measure $\mu^{o}$ on $\overline{\mathcal{A}}$ is invariant under this action. Hence the action of these diffeomorphisms preserves the inner product on $\mathrm{Cyl}^{(3)}$ and extends to a unitary action on all of $\mathcal{H}$. The operators $\hat{V}_{R}$ and $\hat{q}_{x}$ transform covariantly under this action.

There is however a larger group, Diff, of smooth diffeomorphisms of $\Sigma$ that one can consider. Given $\phi \in$ Diff, we obtain an operator on $\mathcal{H}$ whose domain $^{9}$ is the linear span of all cylindrical functions $\Psi_{\gamma}$ such that $\gamma$ is

\footnotetext{
${ }^{9}$ If we work in the Hilbert space of [28] given by all the smooth graphs, then the domain of each diffeomorphism is the whole Hilbert space.
} 
mapped by $\phi$ to an analytically embedded graph. For notational simplicity, we will. denote these operators also by $\phi$. Their action is given by

$$
\phi \Psi_{\gamma}(\bar{A})=\psi\left(\bar{A}\left(\phi\left(e_{1}\right)\right), \ldots, \bar{A}\left(\phi\left(e_{N}\right)\right)\right),
$$

where $\psi$ is the function on $[S U(2)]^{N}$ that determines $\Psi_{\gamma}$ (see (2.3)). The volume operators transform covariantly with respect to these diffeomorphisms. That is,

$$
\begin{aligned}
\phi \hat{V}_{R} \Psi_{\gamma} & =\hat{V}_{\phi(R)} \phi \Psi_{\gamma}, \\
\phi \hat{q}_{x} \Psi_{\gamma} & =\hat{q}_{\phi(x)} \phi \Psi_{\gamma} .
\end{aligned}
$$

In particular if $\phi$ preserves $R$, we have $\left[\phi, \hat{V}_{R}\right]=0$, and, if it preserves $x$, then $\left[\phi, \hat{q}_{x}\right]=0$. Note, however, that the volume operators fail to be covariant with a similar action of an arbitrary homeomorphisrn. In particular, recall that $\hat{V}_{R}$ annihilates $\Psi_{\gamma}$ if the incident edges at every vertex of $\gamma$ are coplanar. However, for every graph there is a homeomorphism that can map it into a graph with the above property, which contradicts the covariance. How does this situation compare with that in the classical theory? Since densities fail to have a meaningful transformation property under general homeomorphisms, the image of the volume element under homeomorphisms may not even exist!

If the region $R$ is all of $\Sigma$, the total volume operator $\hat{V}_{\Sigma}$ is diffeomorphism invariant. Therefore it induces the operator in the space of diffeomorphism invariant states $[9,28])$.

\subsection{The Spectrum}

We will first show that irrespective of the choice of the open region $R$, the volume operators $\hat{V}_{R}$ have the same, discrete spectrum. Note first that, for every cylindrical function $\Psi_{\gamma}, \hat{V}_{R} \Psi_{\gamma}$ as well as $\hat{q}_{x} \Psi_{\gamma}$ are cylindrical over the same graph $\gamma$. Let us therefore fix $\gamma$ and consider the restrictions of (5.1) to $\mathrm{Cyl}_{\gamma}$ which define operators thereon for every region $R \subset \Sigma$ and every point $x \in \Sigma$. In $\mathrm{Cyl}_{\gamma}$ each of the operators $\hat{q}_{v}$ is a firite sum with constant coefficients of the products of triplets of the operators $J_{v, e}^{i}$. It follows from the well known properties of operators satisfying the angular momentum commutation relations (2.6) that the spectrum of $\hat{q}_{v}$ in the completion $\mathcal{H}_{\gamma}$ of $\mathrm{Cyl}_{\gamma}$ is discrete. Therefore, so is the spectrum of the restriction of $\hat{V}_{R}$ to $\mathcal{H}_{\gamma}$ for $\hat{V}_{R}$ is a finite sum of the commuting square roots of $\left|\hat{q}_{v}\right|$ s. If for each $\gamma$ we denote the span of the eigenvectors in $\mathcal{H}_{\gamma}$ by $\mathcal{E}_{\gamma}$ then $\cup_{\gamma} \mathcal{E}_{\gamma}$ is dense in $\mathcal{H}$. Hence the full spectrum of $\hat{V}_{R}$ is given by the union of the spectra on the spaces $\mathcal{H}_{\gamma}$.

If we vary the graph $\gamma$, a priori it is possible that the spectra might change in a continuous manner. However, the spectrum of $\hat{q}_{x}$ depends only on 
the relative orientations of the triplets of oriented tangent directions defined by the edges at $x$. The set of possible characteristics is thus countable. Therefore, the full spectrum of $\hat{q}_{x}$ is a countable union of the countable set of different spectra in each $\mathcal{H} \gamma$. The same argument shows that the full spectrum of $\hat{V}_{R}$ is countable.

The fact that the spectrum of $\hat{V}_{R}$ is independent of an open $R$ is a simple consequence of the fact that the eigenvalues of $\hat{q}_{x}$ depend only on the characteristics of a graph in an arbitrarily small neighborhood of $x$. This property is shared by the classical volume function $V_{R}$ (although in that case the allowed values of the function span the entire non-negative half-line for any open region $R$ ).

It is also clear from the commutation relations (2.6) that there is a basis such that each of the operators $J_{x, e}^{i}$ is of the form $i$ times a skew-symmetric, real and block-diagonal matrix of finite dimensional blocks corresponding to graphs. (This is a spin-network basis $[10,11]$; see also [1] for an extension of the definition of spin-networks from the space of gauge invariant cylindrical functions to the space Cyl of all the cylindrical functions.) Since $\hat{q}_{x}$ is constructed from the products of the commuting $J_{x, e}^{i}$ operators, the same is true for $\hat{q}_{x}$. Therefore, if a real number $\lambda$ is an eigenvalue of $\hat{q}_{x}$ then so is $-\lambda$ and the corresponding eigenvectors are related to each other by complex conjugation of the coefficients.

Given a graph $\gamma$ and a vertex $x, \hat{q}_{x}$ commutes with each of the operators $J_{v, e}^{i}$ provided $v \neq x$ as well as with the Gauss constraint operator

$$
\mathcal{G}_{x}^{i}=\sum_{I=1}^{N} J_{x, e_{I}}^{i},
$$

where $I$ in the sum labels the edges at $x$. From these, one can construct the following set of commuting operators. For each vertex $v \neq x$, number the edges at $v$ in an arbitrary manner, say as $e_{1}, \ldots, e_{N}$, and for every $k \leq N$ define the following operator:

$$
\left(\sum_{I=1}^{k} J_{v, e_{I}}^{i}\right)\left(\sum_{I=1}^{k} J_{v, e_{I}}^{i}\right)=:\left(\sum_{I=1}^{k} J_{v, e_{I}}\right)^{2} .
$$

These operators, together with $\mathcal{G}_{v}^{i_{v}}$ for every vertex $v$ of $\gamma$ and fixed $i_{v}$ for each $v$, form the required commuting set. Subspaces preserved by $\hat{q}_{x}$ can be therefore labelled by the eigenvalues of these operators. Let us denote by $\mathcal{T}_{x, l, j_{1}, \ldots, j_{n}, M}$ the subspace corresponding to the eigenvalue $l(l+1)$ of $\mathcal{G}_{x}^{i} \mathcal{G}_{x}^{i}$, the eigenvalues $j_{I}\left(j_{I}+1\right)$ of $J_{x, e_{I}}^{i} J_{x, e_{I}}^{i}, I$ labeling the edges at $x$ and to $M$ labeling the eigenvalues of the remaining operators.

Whenever $\mathcal{T}_{x, l, j_{1}, \ldots, j_{n}, M}$ is one-dimensional it is necessarily an eigen-direction of $\hat{q}_{x}$. Furthermore, the corresponding eigenvalue must be zero. 
In practice, this is a powerful argument to find the kernel of $\hat{q}_{x}$ and was used by Loll [21] to show that the volume operator must annihilate all gauge invariant $(l=0)$ cylindrical functions $\Psi_{\gamma}$ if all vertices of $\gamma$ are trivalent or have lower valency (i.e., if the number of edges meeting at any vertex is less than or equal to three). If $\mathcal{T}_{x, l, j_{1}, \ldots, j_{n}, M}$ happens to be 2-dimensional, then the absolute value operator $\left|\hat{q}_{x}\right|$ relevant for the volume is automatically diagonal therein. Such a space, namely $\mathcal{T}_{x, \frac{1}{2}, j_{1}, j_{2}, j_{3}, M}$, emerges in the evaluation of the explicit formula for Thiemann's Hamiltonian operator acting on triva,lent spin-networks [26]. In the general case, $\mathcal{T}_{j, l, j_{1}, \ldots, j_{n}, M}$ is finite dimensional, which still reduces the eigen problem to diagonalization of finite dimensional matrices.

The operator $\hat{q}_{x}$ consists of terms of the form

$$
\epsilon_{i j k} J_{e_{I}}^{i} J_{e_{J}}^{j} J_{e_{K}}^{k}=: \hat{q}_{I J K} .
$$

An intriguing property of this expression is that it can be written as

$$
\hat{q}_{I J K}=\frac{1}{4 i}\left[\left(J_{e_{J}}+J_{e_{K}}\right)^{2}\left(J_{e_{I}}+J_{e_{J}}\right)^{2}\right] .
$$

This was observed and used by Thiemann to analyze the matrix of $\hat{q}_{x}$ in a 4 -valent case [22]. That property is also related to the 'apparently anomalous' terms in the commutator of two area operators [25]. Several examples of the eigenvalues and eigenvectors and other special cases were studied in $[17,29,30]$. Although the volume operator studied in [17] differs from ours, and that of [29] is derived within the lattice framework, in the 4- (or lower) valent cases there is a simple relation between all these operators. In particular, the lattice operator coincides with the above operator, restricted to the cylindrical functions given by the loops contained in a cubic lattice [18].

Finally, the spectrum of the volume is discrete in the sense that it has a countable number of elements. The complete spectrum is not known explicitly (in contrast to the situation with area operators). Indeed, we do not even know how 'densely the eigenvalues are packed' on the real line, or even what the smallest non-zero eigenvalue is.

\subsection{Example: A Gauge Invariant 4-Valent Vertex.}

Since vertices at which three or fewer edges meet are 'trivial' as far as the operators $\hat{q}_{x}$ and $\hat{V}_{R}$ are concerned, the simplest non-trivial case is that of a gauge invariant 4 -valent vertex. Therefore, to get a feel for the action of these operators, let us therefore discuss this case in some detail.

Suppose $x$ is a 4 -valent vertex of a graph $\gamma$ and consider $\hat{q}_{x}$ acting in the corresponding subspace of gauge invariant elements of $\mathrm{Cyl}_{\gamma}^{(3)}$. Denote by $e_{I}$, $I=1,2,3,4$, the edges intersecting at a vertex $x$. Gauge invariance of states 
in this subspace is equivalent to the statement that the Gauss constraint vanishes identically on this subspace. Thus, at the vertex $x$ we have:

$$
J_{x, e_{1}}^{i}+J_{x, e_{2}}^{i}+J_{x, e_{3}}^{i}+J_{x, e_{4}}^{i}=0 .
$$

We use this equation to eliminate $J_{x, e_{4}}^{i}$ in favor of the remaining $J_{\mathrm{s}}$. Then, we have

$$
\begin{aligned}
& \hat{q}_{x}=\frac{\kappa_{o}}{8} \epsilon_{i j k}\left(\epsilon\left(e_{1}, e_{2}, e_{3}\right) J_{x, e_{1}}^{i} J_{x, e_{2}}^{j} J_{x, e_{3}}^{k}+\epsilon\left(e_{4}, e_{2}, e_{3}\right) J_{x, e_{4}}^{i} J_{x, e_{2}}^{j} J_{x, e_{3}}^{k}\right. \\
&\left.+\epsilon\left(e_{1}, e_{4}, e_{3}\right) J_{x, e_{1}}^{i} J_{x, e_{4}}^{j} J_{x, e_{3}}^{k}+\epsilon\left(e_{1}, e_{2}, e_{4}\right) J_{x, e_{1}}^{i} J_{x, e_{2}}^{j} J_{x, e_{4}}^{k}\right)
\end{aligned}
$$

Using the Gauss constraint and the observation that

$$
\epsilon_{i j k} J_{x, e_{1}}^{i} J_{x, e_{2}}^{j}\left(J_{x, e_{1}}^{k}+J_{x, e_{2}}^{k}\right)=0,
$$

we find

$$
\hat{q}_{x}=\frac{\kappa_{o}}{8} \kappa\left(e_{1}, e_{2}, e_{3}, e_{4}\right) \epsilon_{i j k} J_{x, e_{1}}^{i} J_{x, e_{2}}^{j} J_{x, e_{3}}^{k}
$$

where

$$
\kappa\left(e_{1}, e_{2}, e_{3}, e_{4}\right)=\epsilon\left(e_{1}, e_{2}, e_{3}\right)-\epsilon\left(e_{1}, e_{2}, e_{4}\right)-\epsilon\left(e_{1}, e_{4}, e_{3}\right)-\epsilon\left(e_{4}, e_{2}, e_{3}\right) .
$$

Thus, modulo the geometric factor $\kappa\left(e_{1}, e_{2}, e_{3}, e_{4}\right)$, the action is the same as that at a trivalent, but non-gauge invariant, vertex between the edges $e_{1}$, $e_{2}$ and $e_{3} .{ }^{10}$ (In a trivalent gauge invariant case, one can further express $J_{x, e_{3}}^{i}$ as $J_{x, e_{3}}^{i}=-J_{x, e_{1}}^{i}-J_{x, e_{2}}^{i}$. Then (5.10) implies that $\hat{q}_{x}$ vanishes on this subspace.)

The value of the diffeomorphism invariant factor $\kappa$ depends on the characteristics of the intersection between the ordered edges at $x$. Recall from Section 4.2 that it depends only on the oriented directions $\dot{e}_{I}, I=1, \ldots, 4$, of the vectors defined by the edges at $x$. If the intersection is planar (that is, the four edges are tangent to a two-plane), then $\hat{q}_{x}$ is identically zero. Otherwise, we can always find coordinates, such that after a possible renumbering,

$$
\dot{e}_{1}=(1,0,0), \dot{e}_{2}=(0,1,0), \dot{e}_{3}=(0,0,1) \text {. }
$$

Then the intersection character is determined by $\dot{e}_{4}$. One can see that every case (modulo renumbering) is diffeomorphic to one of the cases given by the following possible values of $\dot{e}_{4}$ :

$$
\dot{e}_{4}=(1,1,1),(-1,-1,-1),(1,1,0),(-1,-1,0), \dot{e}_{3},-\dot{e}_{3} .
$$

\footnotetext{
${ }^{10}$ It follows from the same arguments that, in a 4-valent case, the Rovelli-Smolin counterpart (A.23) of our $\hat{q}_{x}$ operator (5.11) coincides with $\left|\hat{q}_{x}\right|$ of (5.11) with $\kappa\left(e_{1}, e_{2}, e_{3}, e_{4}\right)=4$.
} 
The corresponding values of $\kappa\left(\epsilon_{1}, e_{2}, e_{3}, e_{4}\right)$ are

$$
\kappa\left(e_{1}, e_{2}, e_{3}, e_{4}\right)=-2,4,-1,3,0,2 .
$$

Finally, the dimension of the corresponding invariant subspace $\mathcal{T}_{x, 0, j_{1}, \ldots, j_{4}, M}$ is given by the number of different numbers $j$ each of which satisfies simultaneously:

$$
\left|j_{1}-j_{2}\right| \leq j \leq\left|j_{1}+j_{2}\right|, \quad\left|j_{3}-j_{4}\right| \leq j \leq\left|j_{3}+j_{4}\right|
$$

and such that both $j-\left|j_{1}-j_{2}\right|$ and $j-\left|j_{3}-j_{4}\right|$ are integers.

\section{Discussion}

In the main body of the paper we presented a regularization scheme to obtain volume operators $\hat{V}_{R}$ associated with open regions $R$ of the 'spatial' 3-manifolds $\Sigma$ and discussed a few properties of these operators. As in any 'quantization', the idea is to first express the classical observable of interest (in our case, functions $V_{R}$ on the classical phase space) in terms of 'elementary' variables (in our case, two-dimensionally smeared triads) which have unambiguous quantum analogs, then promote this 'regulated' classical expression to quantum theory and finally remove the regulators. There is considerable freedom in the first step and we chose an 'internal' regularization in which the volume of an elementary cell is expressed in terms of triads smeared over three two-surfaces passing through the interior of the cell. It was relatively straightforward to carry over the classical expression to the quantum theory and remove the regulators. However, it turned out that the resulting operator carries a memory of the background structures used in the regularization procedure and fails to transform covariantly under diffeomorphisms of $\Sigma$. To rectify this situation, we first averaged the regulated expressions over the 'relevant' background structures and then removed the regulators. The resulting operator $\hat{V}_{R}$ is uniquely defined up to an overall ( $R$-independent) constant $\kappa_{o}$. It is a densely defined, positive, self-adjoint operator and transforms covariantly under the action of $\Sigma$-diffeomorphisms. Its spectrum is purely discrete.

The central part of the paper is contained in Sections 3 and 4, which discuss the intricacies of the regularization procedure. The overall philosophy here is the same as that used in other quantum field theories and the subtleties involved in the continuum limit are of the same nature as those encountered there. In our case, a key simplification occurs because (a large class of) operators on the kinematical Hilbert space $\mathcal{H}$ can be naturally considered as a consistent family of operators on partial Hilbert spaces $\mathcal{H}_{\gamma}$ associated with graphs $\gamma$ [7]. In the present case, in particular, we could 
focus on the restrictions $\hat{V}_{R}^{\gamma}$ of the desired operator $\hat{V}_{R}$ to $\mathcal{H}_{\gamma}$. In the regularization procedure, therefore, we could refer to the graph $\gamma$. The key test of the procedure comes from the consistency requirement: the operators $\hat{V}_{R}^{\gamma}$ so regulated could have failed to be consistent. This did not happen ${ }^{11}$; our family turned out to be consistent and therefore defines a self-adjoint operator $\hat{V}_{R}$ on $\mathcal{H}$.

The regularization procedure could, however, be improved in some respects. First, the regulated expressions $(3.3,3.4)$ are not gauge invariant. However, a cosmetic change can rectify this situation without affecting our arguments or the final result: it suffices to replace the simple integration of $E$ in (3.3) over a two-surface with integration combined with the parallel transport along some fixed paths ending at a fixed point. This additional step does not affect the result which is already gauge invariant. It would be more difficult to make the regularization procedure manifestly diffeomorphism covariant. However, this is largely an aesthetic issue since our final result does enjoy diffeomorphism covariance. Next, at least at first sight, the ambiguity of a multiplicative constant $\kappa_{o}$ appears to be an undesirable feature. Recall however from Section 4.4 that there is another ambiguity at the kinematical level, first pointed out by Immirzi [32] using earlier work of Barbero [31]. This arises from the existence of a canonical transformation which fails to be unitarily implementable and leads to a one-parameter family of unitarily inequivalent representations of the holonomy-triad operators. For volume operators, the net effect is that there is an ambiguity of a multiplicative constant in the spectrum which cannot be eliminated without additional input. Therefore, from a 'practical' viewpoint, the freedom in the choice of $\kappa_{o}$ does not worsen the situation.

The Appendix discusses another regularization scheme, based on constructions given by Rovelli, Smolin and De Pietri $[17,24]$ in the loop representation. This may be called an 'external' regularization because the starting point is an expression of the volume of an elementary cell in terms of triads smeared on the boundary of the cell. In the early discussions, it was believed that this regularization is free of the subtleties we encountered in Section 3.2 while taking the continuum limit in the quantum theory. However, a careful treatment of the limit shows that this is not the case; the assumptions needed to ensure that a well-defined limit exists are completely analogous to those introduced in Section 3.2. The final result does differ from that presented in the main body of the paper. Because of the 'external' regularization, these regulated - and hence also the final - operators do not have

\footnotetext{
${ }^{11}$ In the initial stages, there was some concern because the regularization procedure seemed to be 'state-dependent'. Regarding $\hat{V}_{R}$ as a consistent family of operators $\hat{V}_{R}^{\gamma}$ clarifies the situation: it is as natural to use $\gamma$ to regulate an operator on $\mathcal{H}_{\gamma}$ as it is to use the Fock space structure to normal order operators in Minkowskian quantum field theories.
} 
any information about the details of the tangent vectors to the edges at vertices. Hence they transform covariantly not only under diffeomorphisms but also homeomorphisms on $\Sigma$ in the sense spelled out in Section 5.3. However, in simple situations such as those considered in simplicial treatments [33], the two operators coincide (apart from a constant [18]). Finally, although it is often not explicitly stated, the ambiguity of an overall constant exists also in the 'external' regularization. There, it is buried in one's choice of 'rectangular' cells. If one changes to tetrahedral cells, for example, the overall constant in front of that volume operator would change. Indeed, the situation is parallel to that in the 'internal' approach of the main text. There, the $\kappa_{o}$-ambiguity arose because, while the limit of the classical, averaged expression is insensitive to the choice of the measure used in the averaging, the limit of the quantum operator is unique only up to an overall constant. Similarly, in the 'external' approach, while the limit of the classical, regulated expression is insensitive to the details of the geometry of elementary cells, the corresponding quantum volume operators can differ by an overall constant.

To conclude, we wish to emphasize that the existence of two distinct operators is a reflection only of quantization ambiguities. In both approaches, one begins by re-expressing the classical volume function $V_{R}$ in terms of two-dimensionally smeared triads and takes these 'regulated' classical expressions over to quantum theory. The difference lies in the choice of the 'regulated' classical expressions. In the classical thecry, they both lead to the same function $V_{R}$ when the regulators are removed. In the quantum theory, there is a subtle difference in the corresponding operators $\hat{V}_{R}$.

Acknowledgements. We thank Roberto De Pietri for numerous discussions about the volume operator, and for showing to us the loop assignment which makes the 'external' regularization convergent. One of us (AA) was supported in part by the NSF grant PHY95-14240 and by the Eberly research funds of Penn State. The other (JL) was supported in part by the Alexander von Humboldt-Stiftung $(\mathrm{AvH})$, the Polish Committee on Scientific Research (KBN, grant no. 2 P03B 017 12) and the Foundation for Polish-German cooperation.

\section{Appendix: 'External' or 'Extrinsic' Regularization}

In this Appendix, we will provide a detailed derivation of the analytic formula of the volume operator (reported in [18]) which results from an 'external' regularization scheme. Key ideas behind this regularization are due to Rovelli and Smolin [13]. However, our treatment differs from theirs in some 
ways. First, we work in the connection -rather than loop-representation. Second, as in Section 3, the removal of regulators is a subtle procedure also in this 'external' regularization and the limiting operator can carry a memory of the coordinate system used or may not even exist unless the permissible partitions are restricted suitably. These subtleties, discussed in Subsections A.3 and A.4 below, were overlooked in [13]. Finally, as is the case for the 'internal' regularization discussed in the main text, our 'external' regularization is consistent with the treatment of the Hamiltonian constraint in the literature [14,28]. Let us discuss this point in some detail. Recall first that the domain of this constraint operator is the diffeomorphism invariant subspace of the algebraic dual to the Hilbert space $\mathcal{H}$ [14], or an appropriate extension thereof [28]. The topology one uses to take the limit while removing the regulator is defined as follows. If $\hat{\mathcal{O}}^{\epsilon}$ denotes the regulated operator on $\mathcal{H}$ (where $\epsilon$ symbolizes the regulator) and $\langle S|$ a dual state, then the limit $\hat{\mathcal{O}}$ as $\epsilon \rightarrow 0$ is defined via its action $\langle\hat{\mathcal{O}} S|$ on $\langle S|$ by the following formula

$$
\langle\hat{\mathcal{O}} S \mid \Psi\rangle:=\lim _{\epsilon \rightarrow 0}\left\langle S \mid \hat{\mathcal{O}}^{\epsilon} \Psi\right\rangle,
$$

for every $\Psi \in \mathcal{H}$ in the domain of $\langle S \hat{\mathcal{O}}|$. In the case of the volume operator now under consideration, it appears that the topology used implicitly in [13] is inequivalent to (A.1). (See [34] for the discussion of that topology.) Indeed, if in the derivation presented in [13], or in the one proposed in [22], we take the final limits using the topology corresponding to (A.1), the final result would be different from that given in $[13,17,22]$ (see footnote 13). In our treatment, as in the main text, we will remove the regulator using the Hilbert space topology and obtain a densely defined operator $\hat{V}_{R}$ on $\mathcal{H}$. The dual action of this operator on the dual space coincides with the action defined by (A.1). Thus, while our final answer is equivalent to that of [13], our regularization provides a general procedure which is 'uniformly' applicable to the volume operator as well as the Hamiltonian constraint. Not only is this aesthetically pleasing but quite essential if, for example, one wishes to incorporate the cosmological constant in the Hamiltonian constraint.

As before, one begins with a partition $\mathcal{C}$ of the open region $R$ into cells but associates to each cell $C$ extra paths to parallel transport the triads $E$ to a fixed point. Let us choose an arbitrary partition $\mathcal{C}$ of $R$ into cells. In particular, $\mathcal{C}$ may be the Rovelli-Smolin partition, obtained by fixing coordinates $x^{a}$ covering $R$ and introducing a family of two-surfaces $x^{a}=n \epsilon$, where $a=1,2,3$ and $n$ ranges over the integers, the cells being given by the coordinate cubes. We will see however that to remove the regulator in the quantum theory one cannot just shrink the size of these cells; unless the partition is restricted in a manner similar to that of Section 3 , contributions from some cells may diverge in the limit $\epsilon \rightarrow 0$. Furthermore, even for such restricted partitions, to ensure that the limiting operator is well-defined, one 
has to choose paths in a specific manner, following a prescription given by De Pietri [24]. Roughly speaking, these restrictions specialize the permissible partitions to the 'same extent' that the restrictions of Section 3 do in the 'internal' regularization scheme.

\section{A.1 The Rovelli-Smolin Strategy}

In this sub-section will outline the overall strategy of Rovelli and Smolin using, however, the connection representation.

Let $R$ be an open subset of $\Sigma$ as before. In general, one may not be able to cover $R$ with a single chart. However, the argument presented in the beginning of Section 3 will continue to apply. We can therefore restrict our attention to a topologically trivial region on which a global chart does exist.

As in Section 3.1, let us then cover $R$ with a global coordinate system and let $\mathcal{C}$ be the partition of $R$ into cubic cells. By $\partial C$ we will mean the differential part of the topological boundary of the cell $C \in \mathcal{C}$. For every point $z \in \partial C$ which belongs to (the interior of) a face of $C$ we wish to smear $E$ over $\partial C$ in a gauge covariant manner. For this purpose, to every $z^{\prime} \in \partial C$ let us assign a path $p_{z^{\prime} x_{0}}$ connecting it with a fixed point $x_{0}$ in $C$. For later convenience, we will assume that the path assignment is such that, as we shrink the size of the cell, for every path $p_{z x_{0}}$ we have:

$$
U_{z x_{0}}(A) \rightarrow 1 \in S U(2)
$$

uniformly with respect to $z \in \partial C$, for all the $C \mathrm{~s}$ in $\mathcal{C}$. $A$ cell partition $\mathcal{C}$ with such a path assignment will be denoted by $\tilde{\mathcal{P}}$. Finally, let us introduce a non-negative two-point field $f\left(z, z^{\prime}\right)$ on $\partial C$, which is a density of weight one in $z$ and a scalar in $z^{\prime}$, such that

$$
\int_{\partial C} f\left(z(r, s), z^{\prime}\right) d r d s=1 \text {. }
$$

With this machinery at hand, the covariantly regularized classical momentum $E_{\partial C, f}(z)$ is defined as

$$
E_{\partial C, f}(z)=\frac{1}{2} \int_{\partial C} f\left(z, z^{\prime}\right)\left(U_{p_{z^{\prime} x_{0}}}\right)^{-1} E^{c}\left(z^{\prime}\right) U_{p_{z^{\prime} x_{0}}} \eta_{a b c} d z^{\prime a} \wedge d z^{\prime b} .
$$

The Rovelli-Smolin regularized, squared volume of $C$ is given by ${ }^{12}$

$$
q_{C}\left(E_{i}\right):=\frac{1}{12} \int_{(\partial C)^{3}}\left|\operatorname{Tr}\left(E_{\partial C, f}\left(z_{1}\right) E_{\partial C, f}\left(z_{2}\right) E_{\partial C, f}\left(z_{3}\right)\right)\right| d^{2} z_{1} d^{2} z_{2} d^{2} z_{3},
$$

\footnotetext{
${ }^{12}$ The use of the Rovelli-Smolin loop variables introduces some more freedom in the choice of the extra paths, but that won't be relevant for our arguments. Our aim is to make comparison with [13] easier by writing various formulas in the notation used there.
} 
where by $d^{2} z$ we mean the parametrization dependent area element on $\partial C$ given by any parametrization thereof. Since the gauge transformation law is $E_{\partial C, f}(z) \mapsto g^{-1}\left(x_{0}\right) E_{\partial C, f}(z) g\left(x_{0}\right)$, the trace is gauge invariant. Finally, set

$$
V_{R}^{\tilde{\mathcal{P}}}(A, E):=\sum_{C \in \mathcal{C}} \sqrt{q_{C}(A, E)} .
$$

This is the Rovelli-Smolin regulated volume functional on the classical phase space. As we shrink cells, we have $V_{\tilde{\mathcal{P}}}(A, E) \rightarrow V_{R}(E)$. The idea is again to promote the regulated expression $V_{R}^{\tilde{\mathcal{P}}}$ to the quantum theory and then remove the regulators.

\section{A.2 Regulated Operators}

As in the main paper, we will first focus on a graph $\gamma$ and study the action of the regulated operators on the Hilbert space $\mathcal{H}_{\gamma}$. The overall procedure is parallel to the one adopted in Section 3: the main idea is to replace every $E_{\partial C, f}(z)$ in the functional $q_{C}(A, E)$ by the operator $\hat{E}_{\partial C, f}$.

Let us then begin with an arbitrary partition $\tilde{\mathcal{P}}$ and consider cylindrical functions $\operatorname{Cyl}_{\gamma}(\overline{\mathcal{A}})$ compatible with a graph $\gamma$. We will only assume that the partition is generic with respect to $\gamma$. The operator $\operatorname{Tr}\left(\hat{E}_{\partial C, f}\left(z_{1}\right)\right.$ $\left.\hat{E}_{\partial C, f}\left(z_{2}\right) \hat{E}_{\partial C, f}\left(z_{3}\right)\right)$ acts on elements of $\mathrm{Cyl}_{\gamma}$ through the operators $J_{w_{I}, p_{I}}^{i}$ of (2.5), where $w_{I}$ are the intersection points of the edges $e_{I}$ of $\gamma$ with $\partial C$,

$$
J_{w_{I}}^{i}:=\frac{1}{2} \sum_{p_{I} \text { at } w_{I}} \kappa_{C}\left(p_{I}\right) J_{w_{I}, p_{I}}^{i},
$$

where $p_{I}$ runs over the set of segments of edges of $\gamma$ intersecting $w_{I} \in \partial C$. Let $p_{x_{0} z}$ be the paths in the partition $\tilde{\mathcal{P}}$ and set

$$
U\left(z, z^{\prime}\right):=U_{p_{z x_{0}}} U_{p_{x_{0}, z^{\prime}}} .
$$

Then,

$$
\begin{gathered}
\operatorname{Tr}\left(\hat{E}_{\partial C, f}\left(z_{1}\right) \hat{E}_{\partial C, f}\left(z_{2}\right) \hat{E}_{\partial C, f}\left(z_{3}\right)\right) \psi \\
=\sum_{w_{I}, w_{J}, w_{K} \in \partial C} f\left(z_{1}, w_{I}\right) f\left(z_{2}, w_{J}\right) f\left(z_{3}, w_{K}\right) \\
\cdot \operatorname{Tr}\left(\tau_{i} U\left(w_{I}, w_{J}\right)(A) \tau_{j} U\left(w_{J}, w_{K}\right)(A) \tau_{k} U\left(w_{K}, w_{I}\right)\right) J_{w_{K}}^{k} J_{w_{J}}^{j} J_{w_{I}}^{i}
\end{gathered}
$$

where the points $w_{I}, w_{J}, w_{J}$ on $\partial C$ contribute only if they simultaneously coincide with the isolated intersection points of $\gamma$ with $\partial C$.

Let us denote the right side of of (A.9) by $\hat{q}_{z_{1} z_{2} z_{3}}$. It we could show that it is self-adjoint, then the quantum version $\int d^{2} z_{1} d^{2} z_{2} d^{2} z_{3}\left|\hat{q}_{z_{1} z_{2} z_{3}}\right|$ of (A.5) 
could be well-defined. We will carry out these steps in detail later. It turns out that even when these steps are completed, there are obstacles in removing the regulators. It is convenient to first discuss how they arise and how they can be overcome by appropriately restricting the limiting procedure.

\section{A.3 Ensuring the Convergence I: Restrictions on Cells}

Given $\Psi_{\gamma} \in \mathrm{Cyl}$, it turns out that the shrinking of cells in $\tilde{\mathcal{P}}$ is not sufficient for the vector $V_{R}^{\tilde{\mathcal{P}}} \Psi_{\gamma}$ to converge. Let us isolate the potential problems. Let $\gamma$ be a graph compatible with $\Psi_{\gamma}$. So far, we have only assumed that the partition $\tilde{\mathcal{P}}$ is generic, i.e., that no vertex of $\gamma$ intersects any $\partial C$. (This property can be ensured by assuming that $\tilde{\mathcal{P}}$ is shrunk in such a way that no segment of any cell remains fixed, the assumption being graph $\gamma$ independent.) Now, if we assume that the partition has been sufficiently refined, say by the rescaling the coordinates, the cells of $\tilde{\mathcal{P}}$ can be classified in the following way:

Type (i): $\partial C$ surrounds a vertex of $\gamma$ and intersects every edge which meets that vertex exactly once;

Type (iia): $\partial C$ does not surround any vertex of $\gamma$ and intersects at most one edge of $\gamma$ in at most two points;

Type (iib): $\partial C$ does not surround any vertex of $\gamma$ and intersects more than one edge of $\gamma$.

As the discussion of [13] and Section 3 suggests, it is only the type (i) cells that should contribute non-trivially to the final result. Indeed, for a cell of type (iia),

$$
\hat{q}_{\tilde{\mathcal{P}}} \Psi_{\gamma}=0
$$

because, as we will see below, only $w_{1} \neq w_{2} \neq w_{3} \neq w_{1}$ contribute to (A.9); cells of this type can be ignored. What may be easily overlooked is that one cannot get rid of cells of type (iib) so simply; these contributions can be coordinate dependent and may even diverge. To see this, suppose we just use the partition $\tilde{\mathcal{P}}_{L}$ given by a cubic lattice of the size $L$ defined by the fixed coordinate system on $R$. Consider a case when two edges $e_{1}$ and $e_{2}$ intersecting at $v$ cross the same face of a cell $C_{v}$ containing $v$ (see Fig.3). The other cell $C$ which shares that face is crossed by the edges $e_{1}$ and $e_{2}$. This produces a non-zero term $\hat{q}_{C} \Psi_{\gamma}$. One may, of course, choose another coordinate system such that $e_{1}$ and $e_{2}$ intersect disjoint faces of $C^{\prime}{ }_{v}$ and do not cross the same adjacent cell. But this simply serves to bring out the ambiguity caused by the coordinate dependence. Shrinking of $\tilde{\mathcal{P}}$ does not eliminate that ambiguity, which is sensitive to rotations of a given $C_{v}$ rather than to its size.

For similar reasons, the limiting procedure produces divergences when $e_{1}$ and $e_{2}$ are tangent to each other at $v$. In this case, generically, as one 


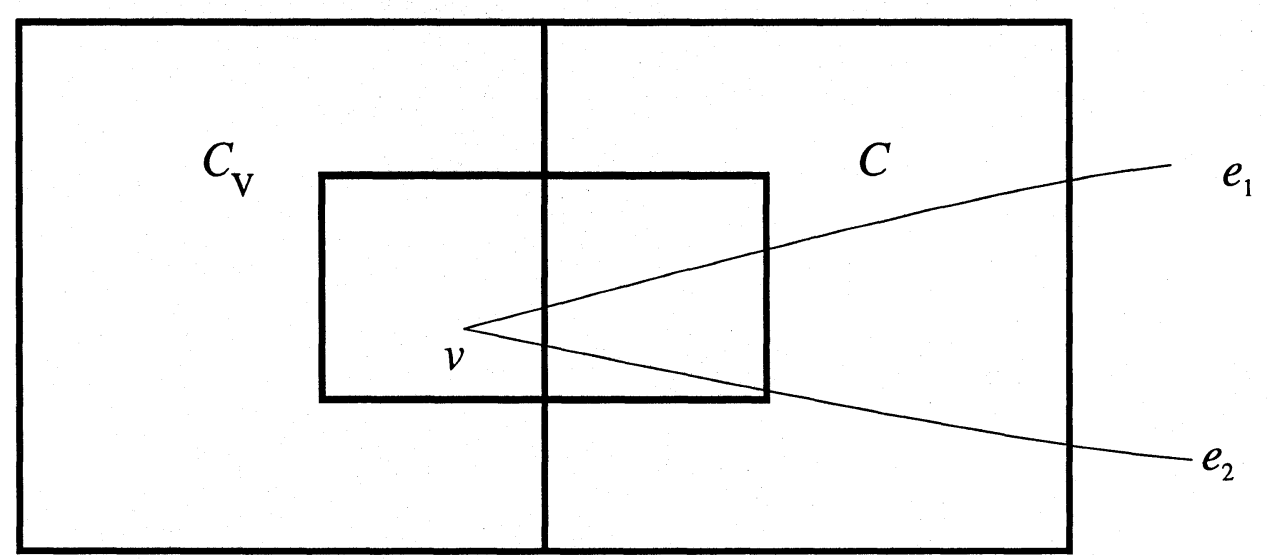

Figure 3: The size change of $C_{v}$ and $C$ does not change the fact that $e_{1}, e_{2}$ intersect the neighboring cell $C$.

shrinks the maximal size of cells, the number of cells crossed simultaneously by $e_{1}$ and $e_{2}$ tends to infinity. One can check that the consequence is that the norm $\left\|\hat{V}_{\mathcal{C}} \Psi_{\gamma}\right\|$ diverges in the limit.

To summarize, to ensure convergence, as in Section 3, one has to put restrictions on how the limit is taken using the graph $\gamma$. The choice we will make - which seems to be the only natural one available - is to restrict ourselves to partitions containing cells only of the types (i) and (iia). This restriction is very similar to that used in Section 3.

\section{A.4 Ensuring the Convergence II: Restrictions on Paths}

For a partition $\tilde{\mathcal{P}}$ satisfying the above restriction, the regulated volume operator is given by

$$
\hat{V}_{R}^{\tilde{\mathcal{P}}} \Psi_{\gamma}=\sum_{v} \sqrt{\hat{q}_{C_{v}}} \Psi_{\gamma}
$$

where $v$ runs through the set of vertices of $\gamma$ and $C_{v}$ is the cell containing $v$. The resulting vector $\hat{V}_{R}^{\tilde{\mathcal{P}}} \Psi_{\gamma}$ still depends on the partition $\tilde{\mathcal{P}}$ through path assignments and its limit as we shrink the cells may still not exist. ${ }^{13}$ Indeed, for a given vertex $v$ and generic path assignment in $\tilde{\mathcal{P}}$, the vector $\sqrt{\hat{q}_{C_{v}}} \Psi_{\gamma}$

\footnotetext{
${ }^{13}$ In the original derivation [13] of this operator, the limit is taken [34] by setting $\lim \sqrt{\ldots}:=\sqrt{\lim \ldots}$, the limit under the square root being obtained by simply setting the holonomies corresponding to the shrunk paths to the identity matrix. Note however that diffeomorphism invariant dual states $\langle S|$ in (A.1) are not sensitive to the shrinking of paths, so they never disappear as far as the action of such states is concerned. This means that if one wishes to use the same topology (A.1) as in the definition of the Hamiltonian constraint operator, the final limit would be different from the one reported in [13].
} 
contains a component ${ }^{14}$ orthogonal to $\sqrt{\hat{q}_{C_{v^{\prime}}}} \Psi_{\gamma}$ corresponding to any vertex $v^{\prime} \neq v$ of $\gamma$. So the problem of convergence reduces to the convergence of each $\sqrt{\hat{q}_{C_{v}}} \Psi_{\gamma}$ as we shrink $C_{v}$ to $v$ separately for every vertex $v$. But if we shrink $C_{v}$ to a cell $C^{\prime}{ }_{v}$ containing $v$ then for a generic paths assignment, the norm $\left\|\sqrt{\hat{q}_{C_{v}}} \Psi_{\gamma}-\sqrt{\hat{q}_{C^{\prime}{ }_{v}}} \Psi_{\gamma}\right\|$ is non-zero, independently of $C^{\prime}{ }_{v}$. The main reason is that $\sqrt{\hat{q}_{C_{v}}} \Psi_{\gamma}$ has a non-zero component on a graph $\gamma_{1}$ obtained from $\gamma$ by introducing new vertices made at the intersection points $w_{I}$ with $\partial C$ and adding new edges, the paths $p_{w_{I} x_{0}}$. The graph $\gamma_{1}^{\prime}$ corresponding to $\sqrt{\hat{q}_{C^{\prime \prime}}{ }} \Psi_{\gamma}$ is (generically) different from $\gamma_{1}$. Moreover, the projection of $\sqrt{\hat{q}_{C^{\prime}{ }_{v}} \Psi_{\gamma}}$ on the space corresponding to $\gamma_{1}^{\prime}$ is, generically, diffeomorphism equivalent to the projection of $\sqrt{\hat{q}_{C_{v}}} \Psi_{\gamma}$ on the space corresponding to $\gamma_{1}$. Hence shrinking does not affect the norm of the projection. All this makes the sequence of vectors $V_{\tilde{\mathcal{P}}} \Psi_{\gamma}$ highly non-convergent as $\tilde{\mathcal{P}}$ is refined.

These problems obviously disappear if, given a graph $\gamma$, we choose a paths assignment such that for the above graphs we have

$$
\gamma_{1}=\gamma_{1}^{\prime}=\gamma
$$

for every cell. Such an assignment does exist and was pointed out to us by De Pietri [17]. It is defined as follows. Denote the edges of $\gamma$ that meet at $v$ by $e_{I}, I=1, \ldots, k$, and orient them to be outgoing. Denote by $e_{I}^{-}$ the segment of $e_{I}$ contained inside $C_{v}$ and by $e_{I}^{+}$the remaining segment of $e_{I}$. To each intersection point $w_{I} \in e_{I} \cap \partial C_{v}$ set $p_{w_{I} v}:=e_{I}^{-}$and to a point $z \in \partial C_{v}$ which is not intersected by an edge, assign any path $p_{z v}$ (such that the previous regularity assumption (A.2) is satisfied).

With these restrictions on the partition $\tilde{\mathcal{P}}$, the regulator can be removed unambiguously. We will evaluate the limit in the next two sub-sections. For the present, we will just comment on the similarity between the restrictions on partitions used in the two regularization schemes: Given a cell containing a vertex $v$, making all the paths begin right at $v$ is the 'external' regularization counter part of the 'internal' regularization assumption that the 2-surfaces $S_{a}$ in $C$ intersect exactly at $v$.

To conclude this discussion let us note that the issue of path assignments did not arise in the 'internal' regularization because we worked not on the space $\overline{\mathcal{A}} / \overline{\mathcal{G}}$ of generalized connections modulo gauge transformations but on the space $\overline{\mathcal{A}}$ of generalized connections themselves. The loop representation used in [13], on the other hand, deals only with $\overline{\mathcal{A}} / \overline{\mathcal{G}}$ and the extra paths become necessary to ensure gauge invariance of smeared triads. In the connection representation, there is a choice: We could have avoided the issue of path dependence entirely by carrying out the 'external' regularization on $\overline{\mathcal{A}}$. We did not do so to keep as close to the treatment of [13] as possible.

\footnotetext{
${ }^{14}$ Here 'component' refers to the decomposition of $\operatorname{Cyl}(\overline{\mathcal{A}})$ iato orthogonal subspaces associated to distinct graphs given by spin-networks [1,9-11].
} 


\section{A.5 The Evaluation}

Let us now return to (A.9) and investigate the operator $\operatorname{Tr}\left(\hat{E}_{\partial C, f}\left(z_{1}\right)\right.$ $\left.\hat{E}_{\partial C, f}\left(z_{2}\right) \hat{E}_{\partial C, f}\left(z_{3}\right)\right)$. One can show that only the terms $w_{1} \neq w_{2} \neq w_{3} \neq w_{1}$ contribute to the sum which is relevant for the self-adjointness of the entries of $|\ldots|$. To see this, note that, in terms of the segments $e_{I}^{-}, e_{I}^{+}$of $\gamma$ that meet at an intersection point $w_{I} \in \partial C$, we have:

$$
J_{w_{I}}^{i}=\frac{1}{2}\left(J_{e_{I}^{+}}^{i}-J_{e_{I}^{-}}^{i}\right) .
$$

In particular, restricted to $\operatorname{Cyl}_{\gamma}(\overline{\mathcal{A}})$, the operators associated with $w_{I}$ commute,

$$
\left[J_{w_{I}^{i}}, J_{w_{I}^{j}}\right]=0 .
$$

Hence the term corresponding to $w_{I}=w_{J}$

$$
\operatorname{Tr}\left(\tau_{i} \tau_{j} U_{w_{I} w_{K}} \tau_{k} U_{w_{K} w_{I}}\right) J_{w_{K}}^{k} J_{w_{I}}^{j} J_{w_{I}}^{i}
$$

vanishes because the trace is antisymmetric in $i, j$.

Let us now suppose that the partition $\tilde{\mathcal{P}}$ satisfies the restrictions of the last two sub-sections. Using De Pietri's path assignment, denote the parallel transports along $e_{I}, e_{I}^{-}$and $e_{I}^{+}$by $U_{I}, U_{I}^{-}$and $U_{I}^{+}$respectively. The action of $J_{w_{I}}^{i}$ on a cylindrical function $\Psi_{\gamma}(A)=\psi\left(U_{1}(A), \ldots, U_{n}(A)\right)$ is given by

$$
J_{w_{I}}^{i} \Psi_{\gamma}(A)=\left(U_{I}^{+}(A) \tau_{i} U_{I}^{-}(A)\right)_{B}^{A} \frac{\partial \psi}{\partial U_{I B}^{A}} .
$$

So that Eq (A.9) now reads

$$
\begin{aligned}
& \operatorname{Tr}\left(\hat{E}_{\partial C, f}\left(z_{1}\right) \hat{E}_{\partial C, f}\left(z_{2}\right) \hat{E}_{\partial C, f}\left(z_{3}\right)\right) \Psi_{\gamma}(A) \\
&= \sum_{w_{I} \neq w_{J} \neq w_{K} \neq w_{I} \in \partial C} f\left(z_{1}, w_{I}\right) f\left(z_{2}, w_{J}\right) f\left(z_{3}, w_{K}\right) \\
& \cdot \operatorname{Tr}\left(\tau_{i} U_{I}^{-}\left(U_{J}^{-}\right)^{-1} \tau_{j} U_{j}^{-}\left(U_{K}^{-}\right)^{-1} \tau_{k} U_{K}^{-}\left(U_{I}^{-}\right)^{-1}\right) \\
& \cdot\left(U_{I}^{+} \tau_{i} U_{I}^{-}\right)_{B}^{A}\left(U_{J}^{+} \tau_{j} U_{J}^{-}\right)_{D}^{C}\left(U_{K}^{+} \tau_{k} U_{K}^{-}\right)_{F}^{E} \\
& \quad \cdot \frac{\partial^{3}}{\partial U_{I B}^{A} \partial U_{J}^{C} \partial U_{K}^{E}} \psi\left(U_{1}(A), \ldots, U_{n}(A)\right) .
\end{aligned}
$$

The evaluation of the right hand side is considerably simplified by the following property which is a consequence of (A.12). The operator $\operatorname{Tr}\left(\hat{E}_{\partial C, f}\left(z_{1}\right)\right.$ $\left.\hat{E}_{\partial C, f}\left(z_{2}\right) \hat{E}_{\partial C, f}\left(z_{3}\right)\right)$ defines a map

$$
\mathrm{Cyl}_{\gamma}^{(3)}(\overline{\mathcal{A}}) \rightarrow \mathrm{Cyl}_{\gamma_{1}}^{(0)}(\overline{\mathcal{A}})
$$


where $\gamma_{1}$ is the graph obtained from $\gamma$ by splitting the edges at the points where they intersect $\partial C$. However, since the operator: is gauge invariant, it preserves the invariance of $\Psi_{\gamma}$ with respect to the gauge transformations at the points of $\partial C$. Therefore,

$$
\operatorname{Tr}\left(\hat{E}_{\partial C, f}\left(z_{1}\right) \hat{E}_{\partial C, f}\left(z_{2}\right) \hat{E}_{\partial C, f}\left(z_{3}\right)\right): \operatorname{Cyl}_{\gamma}^{(3)}(\overline{\mathcal{A}}) \rightarrow \operatorname{Cyl}_{\gamma}^{(0)}(\overline{\mathcal{A}})
$$

That means that the right hand side of (A.17) is in fact a function of the entire parallel transports $U_{I}=U_{I}^{+} U_{I}^{-}$so that one may make the substitution $U_{I}^{-}=1$ and $U_{I}^{+}=U_{I}$ to derive its general form. The result for the action of the operator in the space $\operatorname{Cyl}_{\gamma}(\overline{\mathcal{A}})$ is

$$
\begin{aligned}
& \operatorname{Tr}\left(\hat{E}_{\partial C, f}\left(z_{1}\right) \hat{E}_{\partial C, f}\left(z_{2}\right) \hat{E}_{\partial C, f}\left(z_{3}\right)\right) \\
& \quad=-\frac{1}{4} \sum_{I \neq J \neq K \neq I} f\left(z_{1}, w_{I}\right) f\left(z_{2}, w_{J}\right) f\left(z_{3}, w_{K}\right) \epsilon_{i j k} J_{v e_{I}}^{i} J_{v e_{J}}^{j} J_{v e_{K}}^{k},
\end{aligned}
$$

where $I, J, K$ run through the set labelling the edges of $\gamma$ intersecting at $v$ and, given $I, w_{I}$ is the intersection point of $e_{I}$ with $\partial C$.

\section{A.6 The Integral}

We can now turn to the quantum version of the full formula (A.5). Thus, we want to evaluate

$$
\hat{q}_{C} \Psi_{\gamma}=\frac{1}{12}\left(\int_{(\partial C)^{3}} \operatorname{Tr}\left(\hat{E}_{\partial C, f}\left(z_{1}\right) \hat{E}_{\partial C, f}\left(z_{2}\right) \hat{E}_{\partial C, f}\left(z_{3}\right)\right) d^{2} z_{1} d^{2} z_{2} d^{2} z_{3}\right) \Psi_{\gamma}
$$

for $\Psi_{\gamma} \in \operatorname{Cyl}_{\gamma}^{(3)}(\overline{\mathcal{A}})$. The operator $\hat{E}_{\partial C, f}\left(z_{1}\right) \hat{E}_{\partial C, f}\left(z_{2}\right) \hat{E}_{\partial C, f}\left(z_{3}\right)$ is defined by (A.20) in a subspace $\mathrm{Cyl}_{\gamma}^{(3)}$ for every $\gamma$. It is easy to see that the result agrees on any $\mathrm{Cyl}_{\gamma}^{(3)}(\overline{\mathcal{A}}) \cap \mathrm{Cyl}_{\gamma^{\prime}}^{(3)}(\overline{\mathcal{A}})$ so the operator is consistently defined in $\operatorname{Cyl}(\overline{\mathcal{A}})$. Due to the self-adjointness of the $J$ operators and the commutativity of operators associated with different edges, the total operator is essentially self-adjoint in $\mathrm{Cyl}^{(3)}(\overline{\mathcal{A}})$. Hence, it is meaningful to take its absolute value.

A priori, the integral of an operator-valued function may not be well defined. However, in our case, the only $z_{1}, z_{2}, z_{3}$ dependence of the integrand comes from the functions $f$ in (A.20). Given a graph $\gamma$ and a cell $C$ choose $f$ s such that for every two edges $e_{I} \neq e_{J}$ intersecting $C$, the supports $\Delta_{I}$ and $\Delta_{J}$ of the functions $f\left(w_{I},.\right)$ and $f\left(w_{J},.\right)$ satisfy $\Delta_{I} \cup \Delta_{J}=\emptyset$. Then in 


$$
\begin{aligned}
\int_{(\partial C)^{3}} & \left|\sum_{I, J, K} \ldots\right| d^{6} z \Psi_{\gamma} \\
= & \sum_{I, J, K} \int_{\Delta_{I}} \int_{\Delta_{J}} \int_{\Delta_{K}} f\left(z_{1}, w_{I}\right) f\left(z_{2}, w_{J}\right) f\left(z_{3}, w_{K}\right) d^{6} z|\ldots| \Psi_{\gamma}
\end{aligned}
$$

where the last factor $|\ldots|$ is $z$ independent and where, due to the normalization property, the $f$ 's integrate out to 1 . Finally, for a cylindrical function $\Psi_{\gamma}$ compatible with a graph $\gamma$ and a cell $C_{v}$ containing a vertex $v$,

$$
\hat{q}_{C_{v}} \Psi_{\gamma}=\frac{1}{48} \sum_{I \neq J \neq K \neq I}\left|\epsilon_{i j k} J_{v e_{I}}^{i} J_{v e_{J}}^{j} J_{v e_{K}}^{k}\right| \Psi_{\gamma},
$$

where $I, J, K$ label the edges at $v$. Now the situation is the same as that we encountered in Section 3: the formula is manifestly invariant with respect to the shrinking of of the cells $C_{v}$. Hence, for any partition $\tilde{\mathcal{P}}$ which satisfies the restrictions stated in the previous sub-sections, the volume operator is given by

$$
V_{R} \Psi_{\gamma}=\sum_{v} \sqrt{\hat{q}_{v}} \Psi_{\gamma}
$$

where the sum extends over all the vertices of the graph. This is the formula that was reported in [18]. The final expression is insensitive to the 'intrinsic' structure of the graph at its vertices and depends only on the 'extrinsic' structure which can be registered at the boundary of sufficiently small cells surrounding the vertices.

\section{References}

[1] A. Ashtekar and J. Lewandowski, Class. Quantum Grav. A14 (1997) 55 .

[2] A. Ashtekar and C. J. Isham, Class. Quantum Grav. 9 (1992) 1433.

[3] A. Ashtekar and J. Lewandowski, "Representation theory of analytic holonomy $C^{\star}$ algebras", in "Knots and quantum gravity", J. Baez, ed, Oxford University Press 1994.

[4] J. Baez, Lett. Math. Phys. 31 (1994) 213; "Diffeomorphism invariant generalized measures on the space of connections modulo gauge transformations", in "Proceedings of the conference on quantum topology", D. Yetter, ed, World Scientific 1994. 
[5] J. Baez and S. Sawin, J. Funct. Analysis, 150 (1997) 1.

[6] A. Ashtekar and J. Lewandowski, J. Math. Phys. 5 (1995) 2170.

[7] A. Ashtekar and J. Lewandowski, J. Geom. Phys. 17 (1995) 191.

[8] J. Mourão and D. Marolf, Commun. Math. Phys. 170 (1995) 583.

[9] A. Ashtekar, J. Lewandowski, D. Marolf, J. Mourão and T. Thiemann, J. Math. Phys. 36 (1995) 6456.

[10] C. Rovelli and L. Smolin, Phys. Rev. D53 (1995) 5743.

[11] J. Baez, Adv. Math. 117 (1996) 253; "Spin networks in non-perturbative quantum gravity", in "The Interface of Knots and Physics", L. Kauffman, ed, American Mathematical Society 1996.

[12] C. Rovelli and L. Smolin, Nucl. Phys. B331 (1990) 80.

[13] C. Rovelli and L. Smolin, Nucl. Phys. B442 (1995) 593.

[14] T. Thiemann, Phys. Lett. B380 (1996) 257.

[15] A. Ashtekar, C. Rovelli and L. Smolin, Phys. Rev. Lett. 69 (1992) 237.

[16] L. Smolin, "Recent Developments in Non-Pertubative Quantum Gravity", in "Quantum Gravity and Cosmology", J. Mercader, J. Solá and R. Verdaguer, eds, World Scientific 1992.

[17] R. De Pietri and C. Rovelli, Phys. Rev. D54 (1996) 2664.

[18] J. Lewandowski, Class. Quantum Grav. 14 (1997) 71.

[19] S. Frittelli, L. Lehner and C. Rovelli, Class. Quantum Grav. 13 (1996) 2921.

[20] A. Ashtekar, Acta Cosmologica XX1-2 (1995) 85.

[21] R. Loll, Nucl.Phys. B460 (1996) 143.

[22] T. Thiemann, "Closed formula for the matrix elements of the volume operator in canonical quantum gravity", gr-qc/9606091.

[23] T. Thiemann, "A length operator for canonical quantum gravity", grqc/9606092.

[24] R. De Pietri, private communication.

[25] A. Ashtekar, A. Corichi and J. Zapata, "Quantum Theory of Geometry III: Non-commutativity of Riemannian Structures", in preparation. 
[26] R. Borissov, R. De Pietri and C. Rovelli, Class. Quantum Grav. 14 (1997) 2793.

[27] T. Thiemann, "QSD V: Quantum gravity as the natural regulator of matter quantum field", gr-qc/9705017.

[28] J. Lewandowski and D. Marolf, "Loop constraints: A habitat and their algebra", gr-qc/9710016.

[29] R. Loll, Nucl. Phys. B500 (1997) 405.

[30] R. De Pietri, Nucl. Phys. Proc. Suppl. 57 (1997) 251; Class. Quantum Grav. 14199714.

[31] F. J. Barbero, Phys. Rev. D51 (1995) 5507.

[32] G. Immirzi, Nucl. Phys. Proc. Suppl. 57 (1997) 65; C. Rovelli and T. Thiemann, "The Immirzi parameter in quantum general relativity", gr-qc/9705059.

[33] A. Barbieri, "Quantum tetrahedra and simplicial spin networks", grqc/9707010.

[34] H. A. Morales-Técotl and C. Rovelli, Phys. Rev. Lett. 72 (1994) 3642; private communication from C. Rovelli to J. L. 